\title{
Guidelines for the Establishment of a Model Neighborhood Electric Vehicle (NEV) Fleet
}

Roberta Brayer

Donald Karner

Kevin Morrow

James Francfort

June 2006

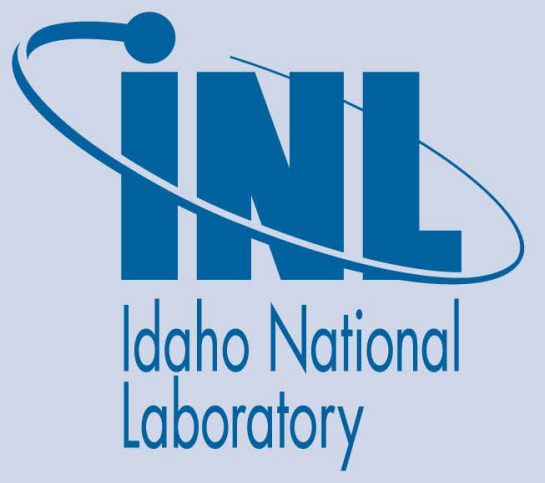

The INL is a U.S. Department of Energy National Laboratory operated by Battelle Energy Alliance 
INL/EXT-06-11309

\title{
Guidelines for the Establishment of a Model Neighborhood Electric Vehicle (NEV) Fleet
}

\author{
Roberta Brayer ${ }^{a}$ \\ Donald Karner \\ Kevin Morrow \\ James Francfort ${ }^{\text {b }}$ \\ ${ }^{\mathrm{a}}$ Electric Transportation Applications \\ ${ }^{\mathrm{b}}$ Idaho National Laboratory \\ June 2006 \\ Idaho National Laboratory
Idaho Falls, Idaho 83415
}

Prepared for the

U.S. Department of Energy

Assistant Secretary for Energy Efficiency and Renewable Energy

Under DOE Idaho Operations Office

Contract DE-AC07-05ID14517 


\section{Disclaimer}

This document highlights work sponsored by agencies of the U.S. Government. Neither the U.S.

Government nor any agency thereof, nor any of its employees, makes any warranty, express or implied, or assumes any legal liability or responsibility for the accuracy, completeness, or usefulness of any information, apparatus, product, or process disclosed, or represents that its use would not infringe privately owned rights. Reference herein to any specific commercial product, process, or service by trade name, trademark, manufacturer, or otherwise does not necessarily constitute or imply its endorsement, recommendation, or favoring by the U.S. Government or any agency thereof. The views and opinions of authors expressed herein do not necessarily state or reflect those of the U.S. Government or any agency thereof. 


\section{ABSTRACT}

The U.S. Department of Energy's Advanced Vehicle Testing Activity tests neighborhood electric vehicles (NEVs) in both track and fleet testing environments. NEVs, which are also known as low speed vehicles, are light-duty vehicles with top speeds of between 20 and $25 \mathrm{mph}$, and total gross vehicle weights of approximately 2,000 pounds or less. NEVs have been found to be very viable alternatives to internal combustion engine vehicles based on their low operating costs. However, special charging infrastructure is usually necessary for successful NEV fleet deployment. Maintenance requirements are also unique to NEVs, especially if flooded lead acid batteries are used as they have watering requirements that require training, personnel protection equipment, and adherence to maintenance schedules.

This report provides guidelines for fleet managers to follow in order to successfully introduce and operate NEVs in fleet environments. This report is based on the NEV testing and operational experience of personnel from the Advanced Vehicle Testing Activity, Electric Transportation Applications, and the Idaho National Laboratory. 


\section{SUMMARY}

Neighborhood Electric Vehicles (NEVs) are defined by the National Highway Traffic Safety Administration as low-speed electric vehicles (Appendix 1) with attainable speeds of more than 20 miles per hour (mph), but not more than $25 \mathrm{mph}$. NEVs are generally allowed to operate on public streets with posted speeds up to $35 \mathrm{mph}$ and are licensed as a motor vehicle.

NEVs are growing in popularity among fleets and the public because of improvements in technology and their inherently low operating costs. In response to this increasing popularity, the U.S. Department of Energy's (DOE) Advanced Vehicle Testing Activity (AVTA) analyzed the potential of NEVs to reduce the use of imported petroleum. ${ }^{\mathrm{c}}$ The subsequent report concluded that NEVs offer the potential to reduce the use of imported petroleum and merit the establishment of testing activities to determine the actual benefits of NEVs and the infrastructure requirements to properly support the operations of NEV fleets. Based on the 2001 Neighborhood Electric Vehicle Fleet Use report, the AVTA (part of DOE's FreedomCar and Vehicle Technologies Program) established several NEV test fleets to collect data and develop information concerning NEV operations in fleet environments.

This report provides a summary of the data collected and lessons learned from NEV testing in various fleets. It also presents the results of analyses of these data in terms of recommending to government, public, and private fleet managers methods and practices to successfully establish NEV fleet operations.

Although NEVs have significant performance limitations, NEV fleet data show that deploying NEVs in properly selected applications with adequate infrastructure can offer the fleet manager functionality at a fraction of the cost of traditional internal combustion engine vehicles. Typical successful applications of NEVs include closed campus environments that limit required operating range and highly structured missions with fixed operating range requirements.

Demonstration fleet data indicates that in addition to mission selection, the fleet manager must address charging infrastructure, vehicle maintenance and training requirements prior to deploying NEVs. The key questions addressed in this report to support successful NEV deployment include:

- What are the requirements of the different vehicle missions within my fleet?

- What NEVs are available on the market and how do I assess what will work best?

- How will NEV maintenance be performed?

- What charging infrastructure will be required?

- What training will be required to support NEV deployment?

- What will capital and operating costs be?

A NEV Fleet Checklist is provided in Appendix 2 for an easy reference tool back to the main document.

This report is based on the NEV testing and operational experience of personnel from the Advanced Vehicle Testing Activity, Electric Transportation Applications, and the Idaho National Laboratory.

c Field Operations Program Report, “Neighborhood Electric Vehicle Fleet Use,” J. Francfort, M. Carroll, July 2001. 


\section{CONTENTS}

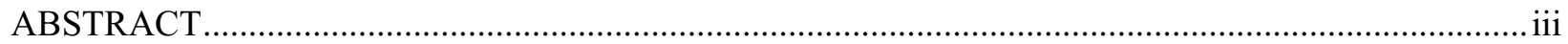

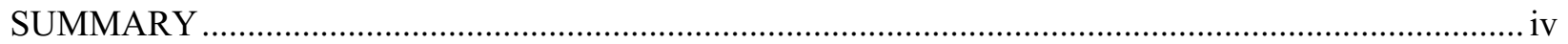

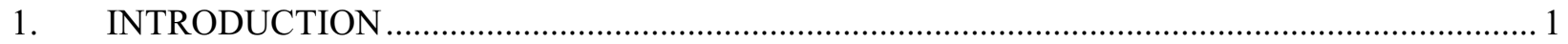

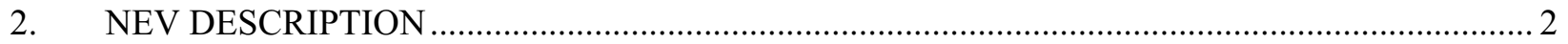

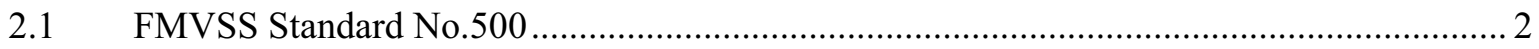

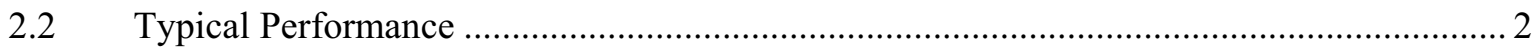

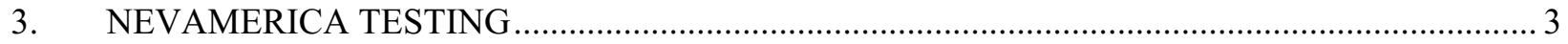

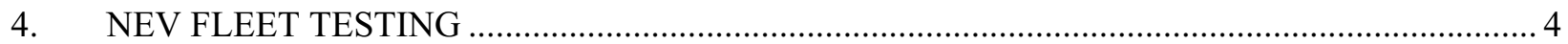

4.1 Luke Air Force Base........................................................................................ 4

4.2 Marine Corps Recruit Depot San Diego ......................................................................... 6

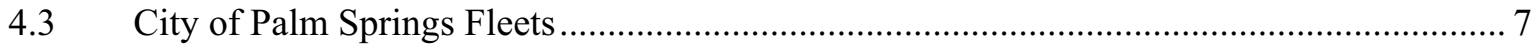

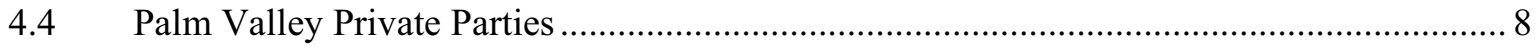

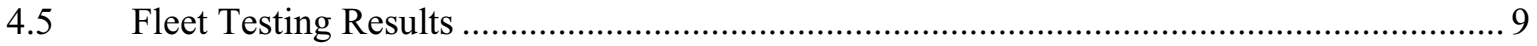

4.5.1 Preventative Maintenance ........................................................................... 10

4.5.2 Corrective Maintenance …………………………………………………….... 11

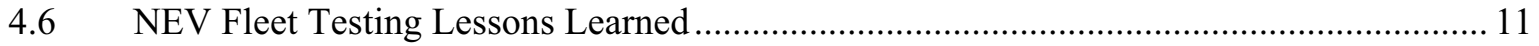

4.6.1 Mission selection............................................................................. 12

4.6.2 Charge infrastructure............................................................................ 12

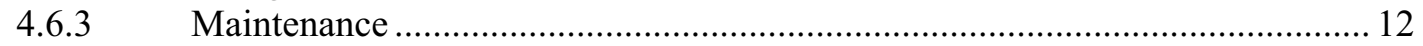

4.6.4 Operator training ………………………………………………………... 12

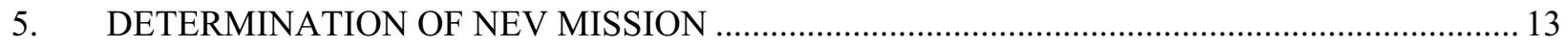

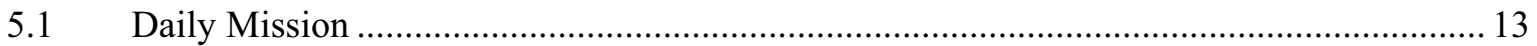

5.1.1 Record Daily Routine Vehicle Miles ……………………………………....13

5.1.2 Note Extraordinary Miles........................................................................... 13

5.1.3 Identify Daily Route Stops........................................................................... 13

5.2 Payload Requirements (Passenger or cargo haulers) ............................................................. 13

5.3 Vehicle Options for Extreme Climates.............................................................................. 14

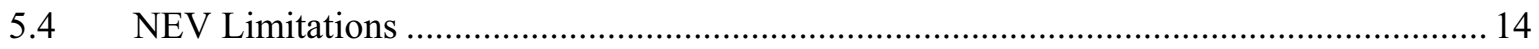

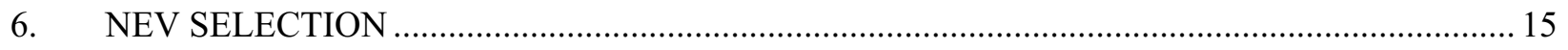

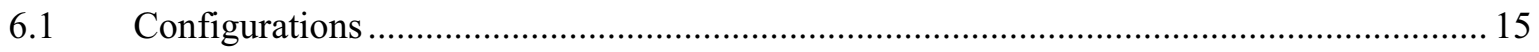

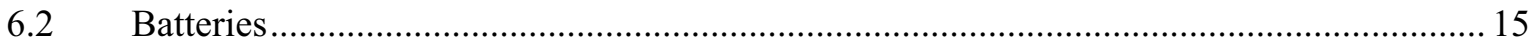

6.2.1 Battery Cost..................................................................................... 16

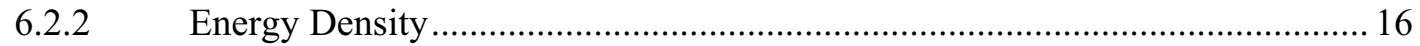

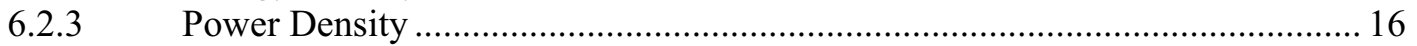

6.2.4 Estimated Cycle Life............................................................................... 16

6.2.5 Charge Retention................................................................................ 17

6.2.6 Fast Charging ………………………………..................................... 17

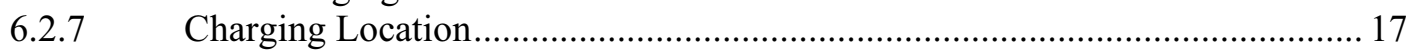

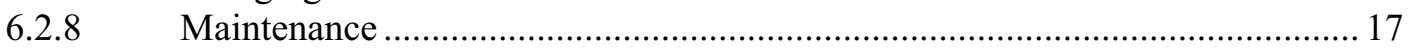




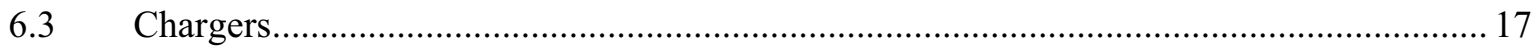

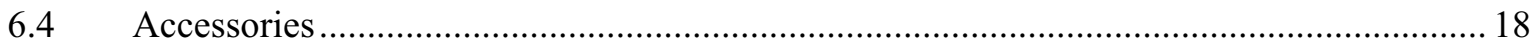

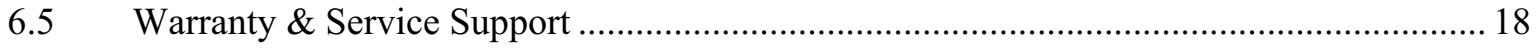

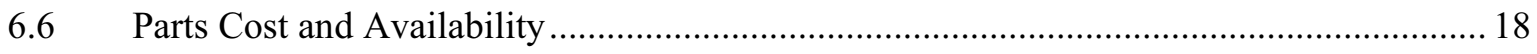

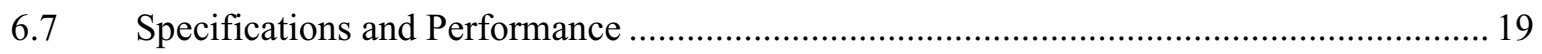

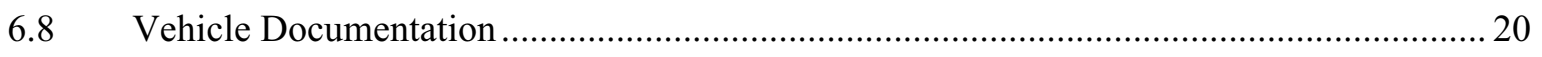

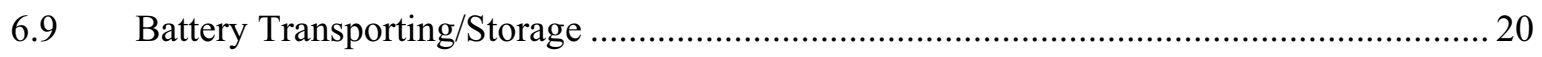

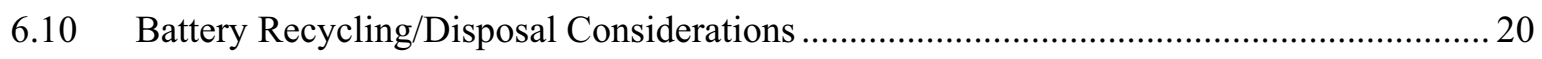

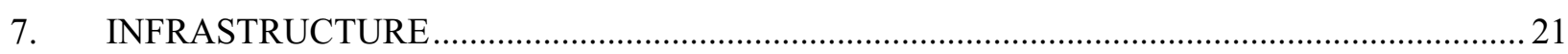

7.1 Charging/Electrical Infrastructure Requirements ................................................. 21

7.2 Maintenance Infrastructure Requirements................................................................. 22

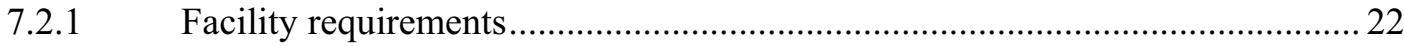

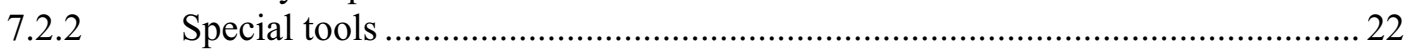

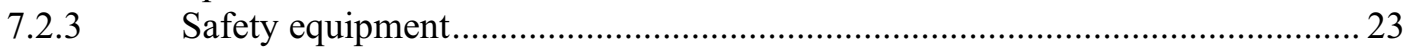

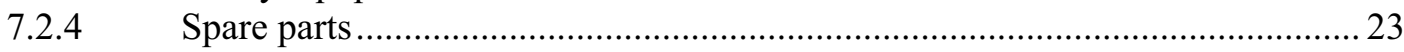

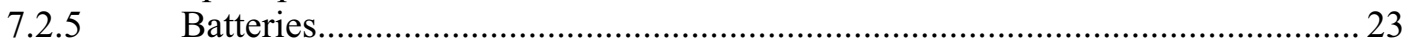

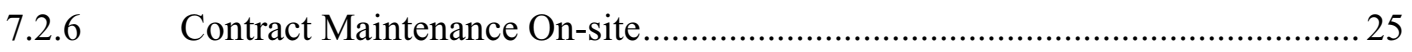

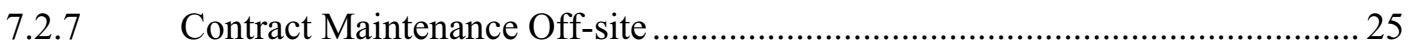

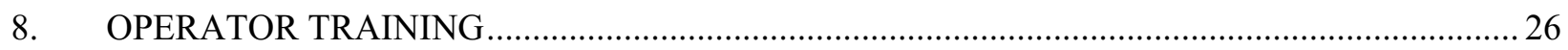

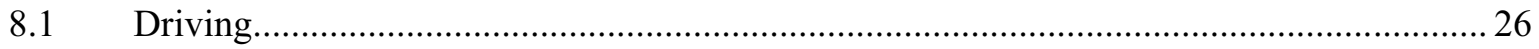

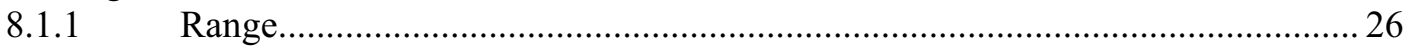

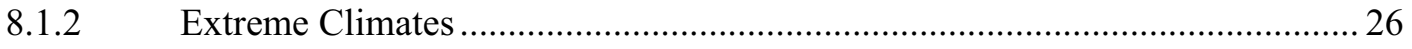

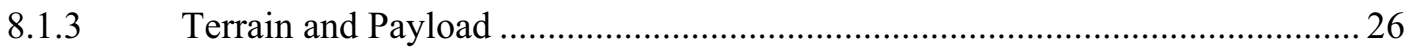

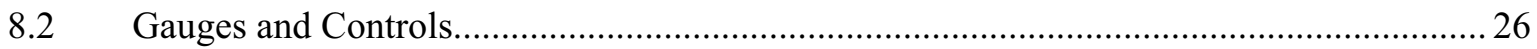

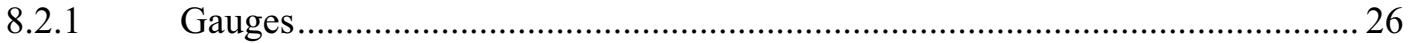

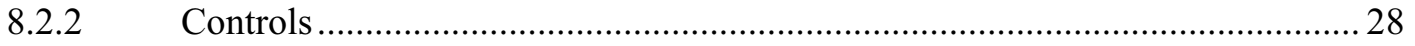

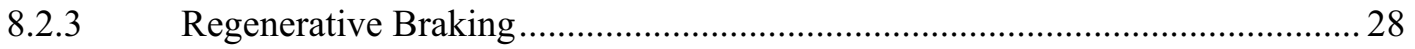

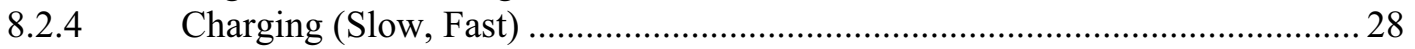

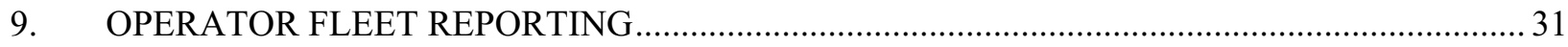

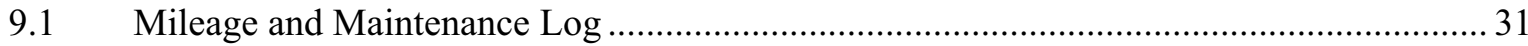

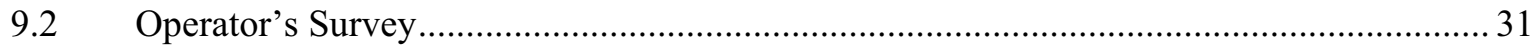

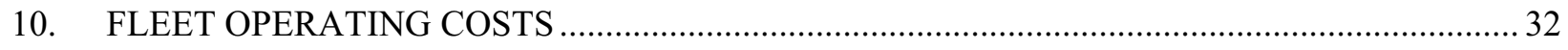

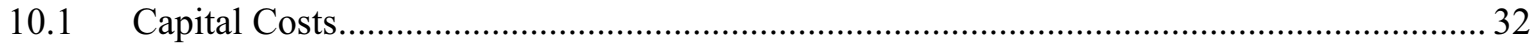

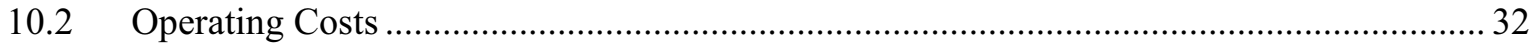

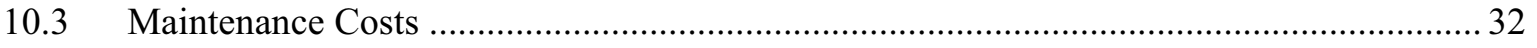

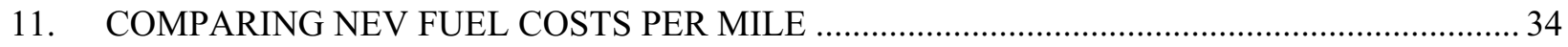




\section{FIGURES}

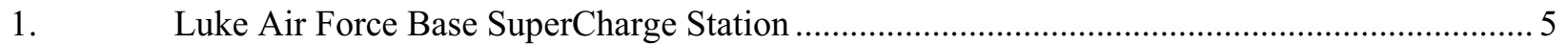

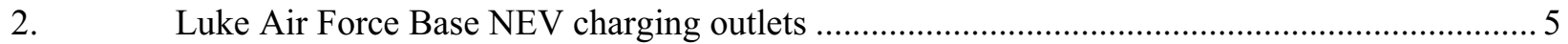

3. NEV parking lot charging at Luke Air Force Base ........................................................... 6

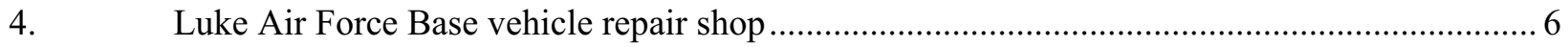

5. LSV-100 NEV charger in the City of Palm Springs' City Hall parking garage ....................... 8

6. City of Palm Springs SuperCharge Station ...................................................................... 8

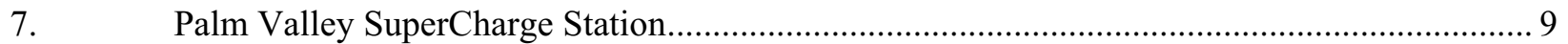

8. Payload for the Columbia ParCar Passenger NEV is 750 pounds ........................................ 14

9. Typical payloads for NEV cargo haulers are up to 1,000 pounds.......................................... 14

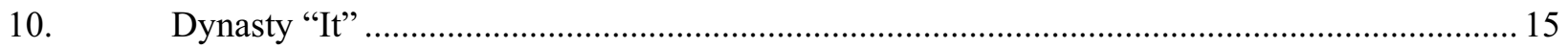

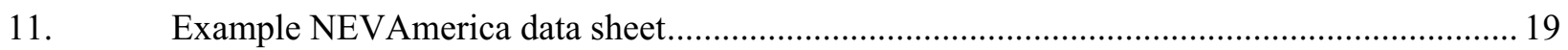

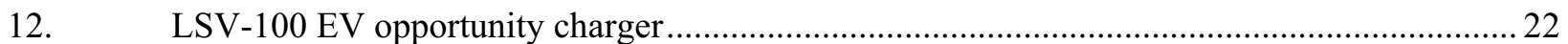

13A-B. GEM NEV dash with turn signal indicators, and an all-in-one digital display for speedometer,

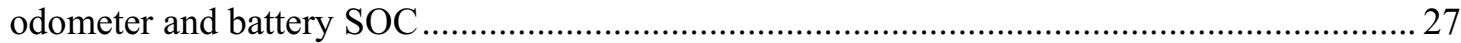

14A-B. Dynasty NEV SOC gauge, directional signals, high-beam indicator, charging indicator and

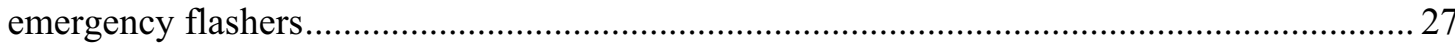

15A-B. Dynasty NEV center dash displaying the speedometer, odometer, gear selection and

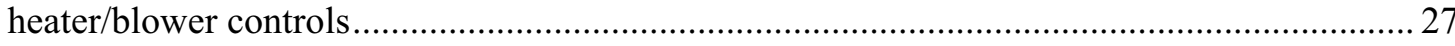

16A-B. GEM — turf/road selection, and forward/reverse selection .............................................. 28

17. $2003 \mathrm{GEM}-120 \mathrm{~V}$ inlet located in front hood, under the GEM emblem..............................2 28

18. 2002 GEM 4-Passenger $120 \mathrm{~V}$ inlet located below the rear passenger side seat..................... 29

19A-B. Dynasty "It" $120 \mathrm{~V}$ inlet located on the driver's side front quarter panel............................... 29

20A-B. GEM fast charge inlet installed on side of front seat support ................................................2 29

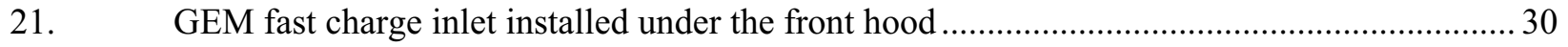

22A-B. Dynasty front fast charge inlet installed behind license plate ............................................. 30

23. Fuel cost per mile for NEVs, hybrid electric vehicles (HEVs), and internal combustion engine (ICE) vehicles. Three NEV electricity costs are used as are two gasoline costs ...................... 35

\section{TABLES}

1. Sample results from 2002 NEV America testing ................................................................. 3

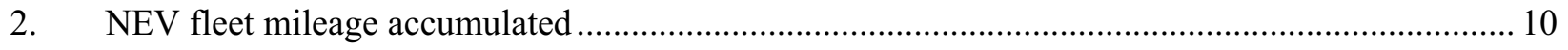

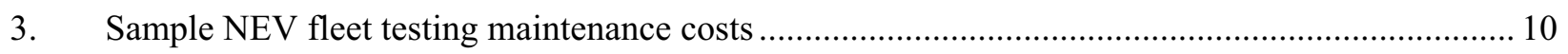

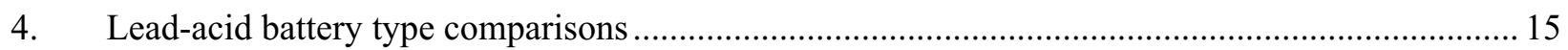

5. NEV energy efficiency as measured at the charger during NEVAmerica baseline performance testing for the twelve NEVs tested with onboard chargers and conductive connectors. 


\section{Guidelines for the Establishment of a Model Neighborhood Electric Vehicle (NEV) Fleet}

\section{INTRODUCTION}

The U.S. Department of Energy's (DOE) Advanced Vehicle Testing Activity (AVTA) conducts neighborhood electric vehicle (NEV) testing to support the AVTA goal of providing benchmark data of emerging technologies for technology modeling, and research and development programs conducted by $\mathrm{DOE}$ and its industry partners. The testing results are also provided to fleet managers and the general public to support their vehicle acquisition processes.

This report supports the fleet manager that has minimal experience operating NEVs, but is considering introducing them into his or her fleet. While a few parts of this report may appear to be critical of the performance and maintenance requirements of NEVs, it is more the intent of the authors to provide a balanced analysis of operating NEVs in fleets. It is important that prospective NEV fleet managers and NEV acquirers be fully "NEV prepared" in order to successfully introduce NEVs to fleets and vehicle users. While most NEV operations are the same as traditional gasoline fueled vehicles (turn a key to start the vehicle, push the turn signal handle down to turn on the left turn signal, etc.), the infrastructure needs and many maintenance needs are NEV specific. For instance, when another gasoline fleet vehicle is acquired, this vehicle would fuel either at the fleet-operated gasoline pump or at a gasoline station down the street. However, adding NEVs usually requires adding the proper charging infrastructure that is very critical to successful NEV deployment. In addition, each fleet manager should check with the appropriate state motor vehicle department for current registration and licensing requirements and laws pertaining to operating NEVs on public streets as these requirements are not uniform in all states.

The AVTA primarily uses two types of testing methods to evaluate advanced technology vehicles. (1) Baseline Performance Testing is conducted on dynamometers and closed test tracks, allowing for a highly controlled testing environment and vehicle-to-vehicle comparisons of the testing results. However, because of the limited top speeds, range, and weight of the NEVs, dynamometer testing is not conducted on NEVs. (2) Fleet Testing places NEVs in government and industry fleets where operating and maintenance data are collected. The fleet testing also provides a very real-world element to the testing process.

It is from the experienced gained from testing NEVs and operating them in fleets that the authors discuss the issues relevant to successfully introduce and operate NEVs in fleet operations.

Other vehicle technologies that the AVTA are currently or will soon be testing include hybrid electric vehicles, hydrogen and hydrogen/compress natural gas blended fuels in internal combustion engine vehicles, and plug-in hybrid electric vehicles. See the AVTA's Webpage (http://avt.inl.gov) for more information on these testing activities.

The AVTA is part of DOE's FreedomCAR and Vehicle Technologies Program. The Idaho National Laboratory and its testing partner, Electric Transportation Applications, of Phoenix, Arizona, conduct the AVTA testing activities discussed in this report. 


\section{NEV DESCRIPTION \\ 2.1 FMVSS Standard No.500}

Because of the increased number of golf carts and similar-sized vehicles that were being used on neighborhood streets and locations like retirement and planned communities, the National Highway Traffic Safety Administration adopted a final NEV rule on June 17, 1998. This new rule, Federal Motor Vehicle Safety Standard (FMVSS) No. 500, is codified in 49 CFR 271.500 and defines a new type of vehicle, the "low-speed vehicle" (LSV). FMVSS No. 500 includes the safety requirements associated with such a vehicle. LSVs are defined as a 4-wheeled motor vehicle, whose attainable speed is more than $20 \mathrm{mph}$ and not more than $25 \mathrm{mph}$. LSVs require basic safety features such as seat belts, turn signals, headlights and taillights, and windshield wipers. A full copy of FMVSS 500 is included in Appendix 1. LSVs are commonly referred to as NEVs.

\subsection{Typical Performance}

NEVS are designed to meet most light-duty applications, such as people movers and light utility use. NEVs are significantly faster than golf carts, which typically have top speeds of 12 to $15 \mathrm{mph}$. Typical NEV payload capabilities range from 600 pounds to 1,000 pounds (including passengers). When the batteries are functioning properly, a fully functional range is typically around 30 miles for each full charge in mild climates. In cold climates, the range can be reduced by as much as half. Options are available, such as fast charging, that allow the range to be extended to over 100 miles per day by opportunity charging in 20 to 30 -minute increments throughout the day. 


\section{NEVAMERICA TESTING}

The AVTA has conducted baseline performance testing of fourteen NEVs through its NEVAmerica program. Baseline performance testing evaluates several areas of vehicle performance, including vehicle range, efficiency, acceleration, handling, and maximum speed. In 2002, the AVTA tested 10 NEVs from Global Electric Motorcars (GEM), Ford Motor Co., Frazer-Nash, and Columbia ParCar. In 2005, another four NEVs from GEM were tested. Copies of the performance data sheets are provided in Appendix 3, and sample results are shown in Table 1. For the complete set of testing specifications and procedures, see the AVTA's NEVAmerica testing results at: http://avt.inl.gov/nev.shtml.

Table 1. Sample results from 2002 NEVAmerica testing.

\begin{tabular}{|c|c|c|c|c|c|}
\hline & $\begin{array}{c}\text { Max. } \\
\text { Speed }^{\text {a }}\end{array}$ & $\begin{array}{l}\text { Range at } \\
\text { Max. } \\
\text { Speed }^{\text {b }}\end{array}$ & $\begin{array}{l}\text { Stopping } \\
\text { Distance }^{c}\end{array}$ & $\begin{array}{l}\text { Max. Speed } \\
\text { on } 6 \% \\
\text { Grade }\end{array}$ & $\begin{array}{c}\text { Charge } \\
\text { Time }^{\mathrm{d}}\end{array}$ \\
\hline MY05 GEM Long Bed Utility & $22.7 \mathrm{mph}$ & 40.6 miles & $33 \mathrm{ft}$ & $19.2 \mathrm{mpg}$ & $6.7 \mathrm{hr}$ \\
\hline MY05 GEM Short Bed Utility & $24.1 \mathrm{mph}$ & 36.7 miles & $27 \mathrm{ft}$ & $24.1 \mathrm{mph}$ & $6.0 \mathrm{hr}$ \\
\hline MY05 GEM (2-Passenger) & $23.2 \mathrm{mph}$ & 44.3 miles & $34 \mathrm{ft}$ & $19.6 \mathrm{mph}$ & $6.9 \mathrm{hr}$ \\
\hline MY05 GEM (4-Passenger) & $24.0 \mathrm{mph}$ & 40.5 miles & $31 \mathrm{ft}$ & $19.4 \mathrm{mph}$ & $6.9 \mathrm{hr}$ \\
\hline MY02 Columbia ParCar (2-Passenger) & $21.6 \mathrm{mph}$ & 52.9 miles & $19 \mathrm{ft}$ & $13.9 \mathrm{mph}$ & $11.3 \mathrm{hr}$ \\
\hline MY02 Columbia ParCar (4-Pass) & $23.3 \mathrm{mph}$ & 47.1 miles & $21 \mathrm{ft}$ & $13.1 \mathrm{mph}$ & $12.0 \mathrm{hr}$ \\
\hline MY02 Ford Th!nk Neighbor (2-Pass.) & $24.2 \mathrm{mph}$ & 33.1 miles & $20 \mathrm{ft}$ & $17.0 \mathrm{mph}$ & $8.3 \mathrm{hr}$ \\
\hline MY02 Ford Th!nk neighbor (4-Pass.) & $24.1 \mathrm{mph}$ & 38.6 miles & $22 \mathrm{ft}$ & $18.3 \mathrm{mph}$ & $8.3 \mathrm{hr}$ \\
\hline MY02 GEM Long Bed Utility & $23.9 \mathrm{mph}$ & 36.2 miles & $22 \mathrm{ft}$ & $17.6 \mathrm{mph}$ & $12 \mathrm{hr}$ \\
\hline MY02 GEM Short Bed Utility & $21.3 \mathrm{mph}$ & 41.2 miles & $24 \mathrm{ft}$ & $17.3 \mathrm{mph}$ & $12 \mathrm{hr}$ \\
\hline MY02 GEM (2-Passenger) & $23.7 \mathrm{mph}$ & 33.4 miles & $22 \mathrm{ft}$ & $16.8 \mathrm{mph}$ & $12 \mathrm{hr}$ \\
\hline MY02 GEM (4-Passenger) & $23.7 \mathrm{mph}$ & 31.4 miles & $20 \mathrm{ft}$ & $18.0 \mathrm{mph}$ & $12 \mathrm{hr}$ \\
\hline $\begin{array}{l}\text { MY01 Frazer-Nash City Car } \\
\text { (4-Passenger) }\end{array}$ & $24.6 \mathrm{mph}$ & 30.9 miles & $17 \mathrm{ft}$ & $23.1 \mathrm{mph}$ & $56^{\mathrm{e}} \mathrm{min}$ \\
\hline MY01 Frazer-Nash 4XSLSV & $24.7 \mathrm{mph}$ & 32.7 miles & $18 \mathrm{ft}$ & $24.5 \mathrm{mph}$ & $58^{\mathrm{f}} \min$ \\
\hline \multicolumn{6}{|c|}{$\begin{array}{l}\text { b. The range is determined by operating the NEV on a closed track under the "brick" test - the accelerator pedal is held to the } \\
\text { floor and the NEV is operated until it can no longer maintain an average lap speed of } 19 \mathrm{mph} \text {. There is an exception for the } \\
\text { average lap speed, depending on the test track used. For the complete range testing methodology, see ETA-NTP004 Electric } \\
\text { Vehicle Constant Speed Range Test at: http://avt.inl.gov/pdf/nev/ntp004.pdf }\end{array}$} \\
\hline $\begin{array}{l}\text { c. From } 20 \mathrm{mph} \text { on dry pavement } \\
\text { d. Using onboard } 120 \mathrm{~V} \text { charger } \\
\text { e. Offboard Level } 3 \text { fast charger, time in minutes } \\
\text { f. Offboard Level } 3 \text { fast charger }\end{array}$ & & & & & \\
\hline
\end{tabular}




\section{NEV FLEET TESTING}

The AVTA accumulated 168,000 fleet testing miles on 101 NEVs both in five government fleets and when operated by private parties. The fleet testing results are discussed below by fleet.

\subsection{Luke Air Force Base}

Luke Air Force Base (LAFB) is located 20 miles west of Phoenix, Arizona. The primary mission of LAFB is F-16 pilot training. Approximately 200 fighter aircraft are based at LAFB. The facility encompasses 4,200 acres. The maximum speed on the base is $25 \mathrm{mph}$, making it an ideal application for NEVs.

LAFB is home to numerous training squadrons, each with its own command structure. Prior to participation in AVTA fleet testing, LAFB command ordered that small electric vehicles (golf carts and NEVs) would be owned by each individual squadron, rather than provided by a central motor pool as is done with full-size vehicles. Therefore, no single fleet manager was responsible for the operation of the NEVs at LAFB.

AVTA data collection was coordinated with the LAFB environmental group, which monitors all electric vehicle activity for environmental reporting purposes. Having multiple squadrons at LAFB, managing their individual NEV resources, emulates multiple small autonomous fleets operating without an automotive fleet manager. This situation would be typical of a small to medium size business in which transportation was only incidental to the business operations.

A total of 55 NEVs were tracked in the LAFB fleet and GEM was the manufacturer of all of these vehicles. Twenty-one of the NEVs were equipped to operate from a fast charger installed at a centrally located parking lot (Figure 1). Each squadron determined the necessary charge infrastructure needed to support its NEV fleet. Typically, this consisted of 120 Volt (V) alternating current (AC) single phase outlets installed at NEV parking locations (Figure 2). However, many times it consisted of the use of long extension cords reaching across an existing parking lot. Late into AVTA fleet testing at LAFB, base command established a NEV parking lot equipped with $120 \mathrm{~V}$ electrical outlets (Figure 3). This parking lot was intended to allow overnight charging of the NEVs at LAFB regardless of squadron assignment.

Mission selection was the prerogative of the individual squadron or department funding the NEV purchase, and no mission guidelines were provided other than the typically optimistic claims of range, charge time, and maintenance requirements provided by vehicle salesmen. Missions were typically categorized as on-base personnel transportation in a "motor pool" type environment. A few specific missions (e.g., flight line fire suppression system maintenance) were employed that often required vehicle modifications for hauling and/or towing equipment required for that assignment.

Maintenance was also left up to the individual funding the NEV purchase. A GEM dealer located in northwest Phoenix, approximately 20 miles from LAFB, typically performed maintenance. At various times throughout AVTA fleet testing at LAFB, NEV maintenance was also available at the base "auto shop" (Figure 4). 


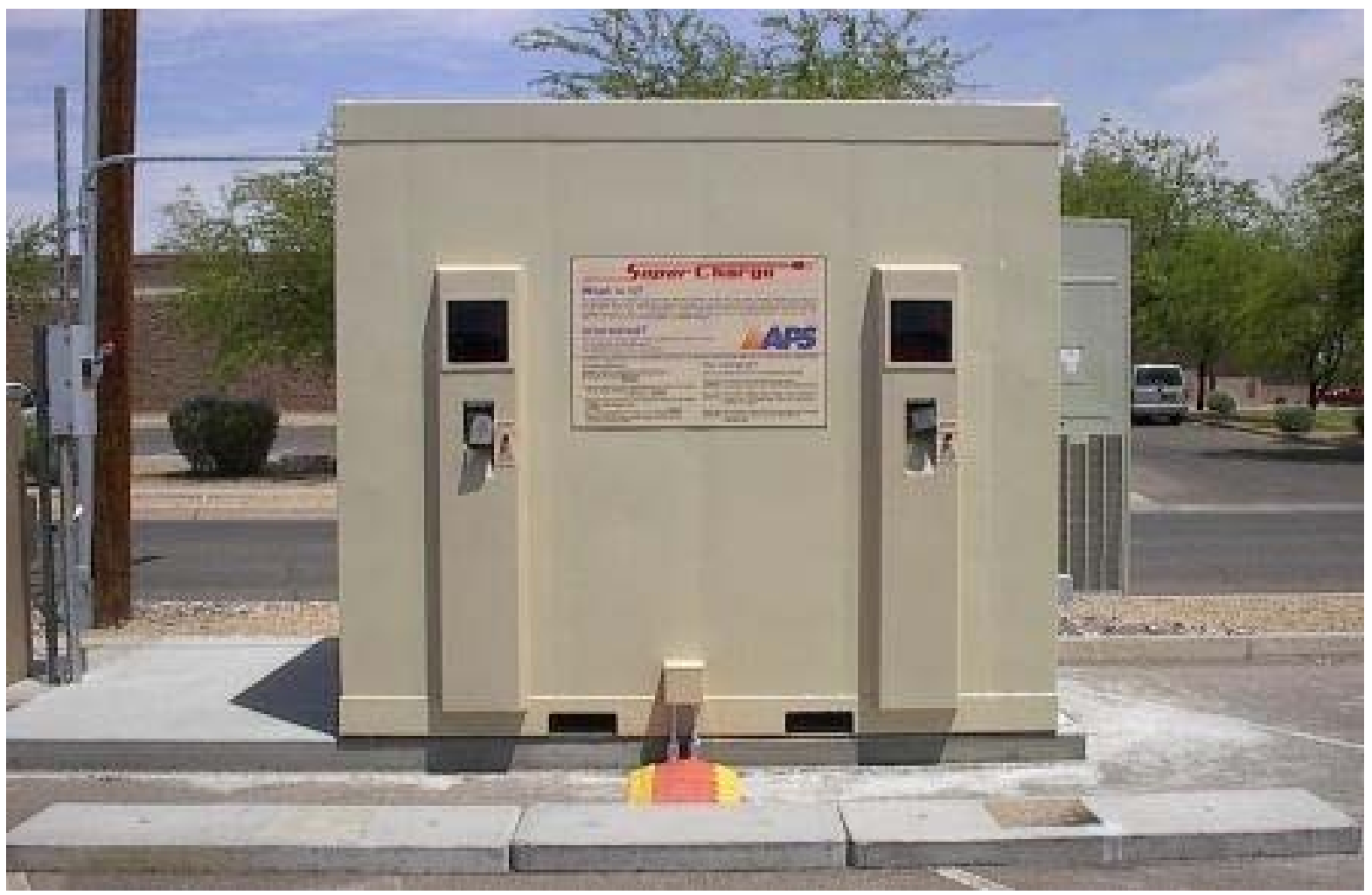

Figure 1. Luke Air Force Base SuperCharge Station.

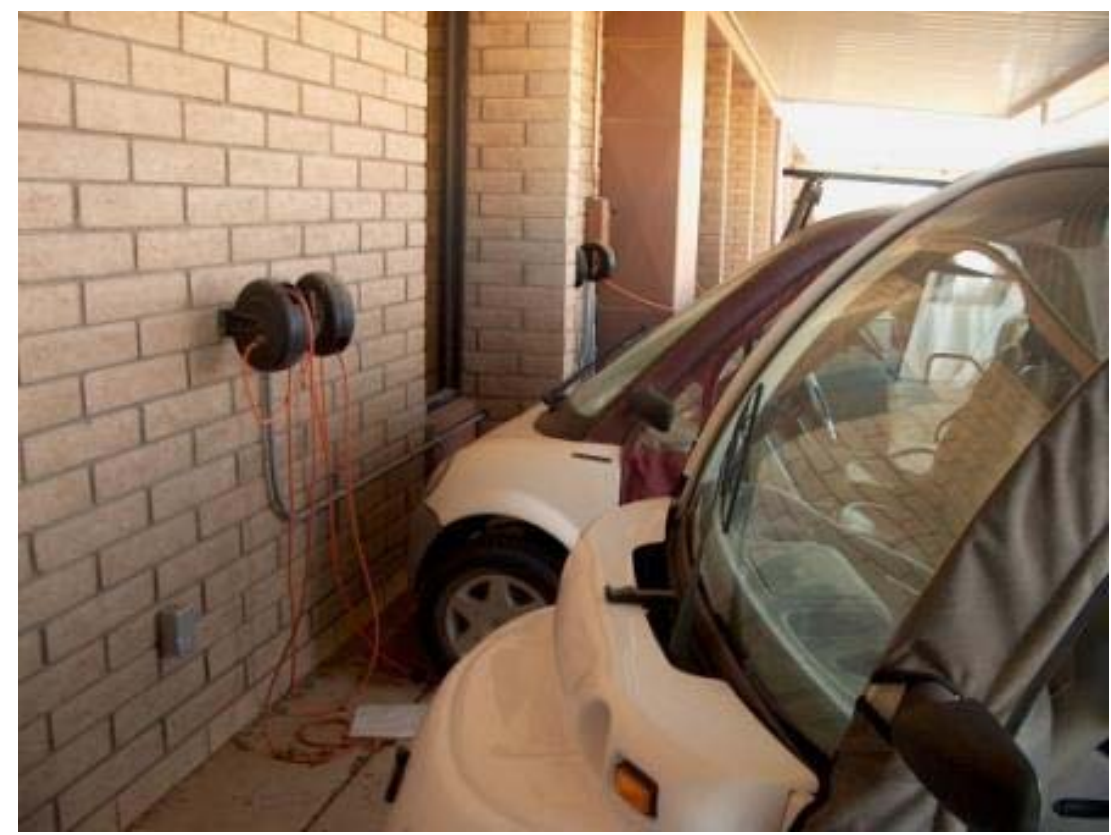

Figure 2. Luke Air Force Base NEV charging outlets. 


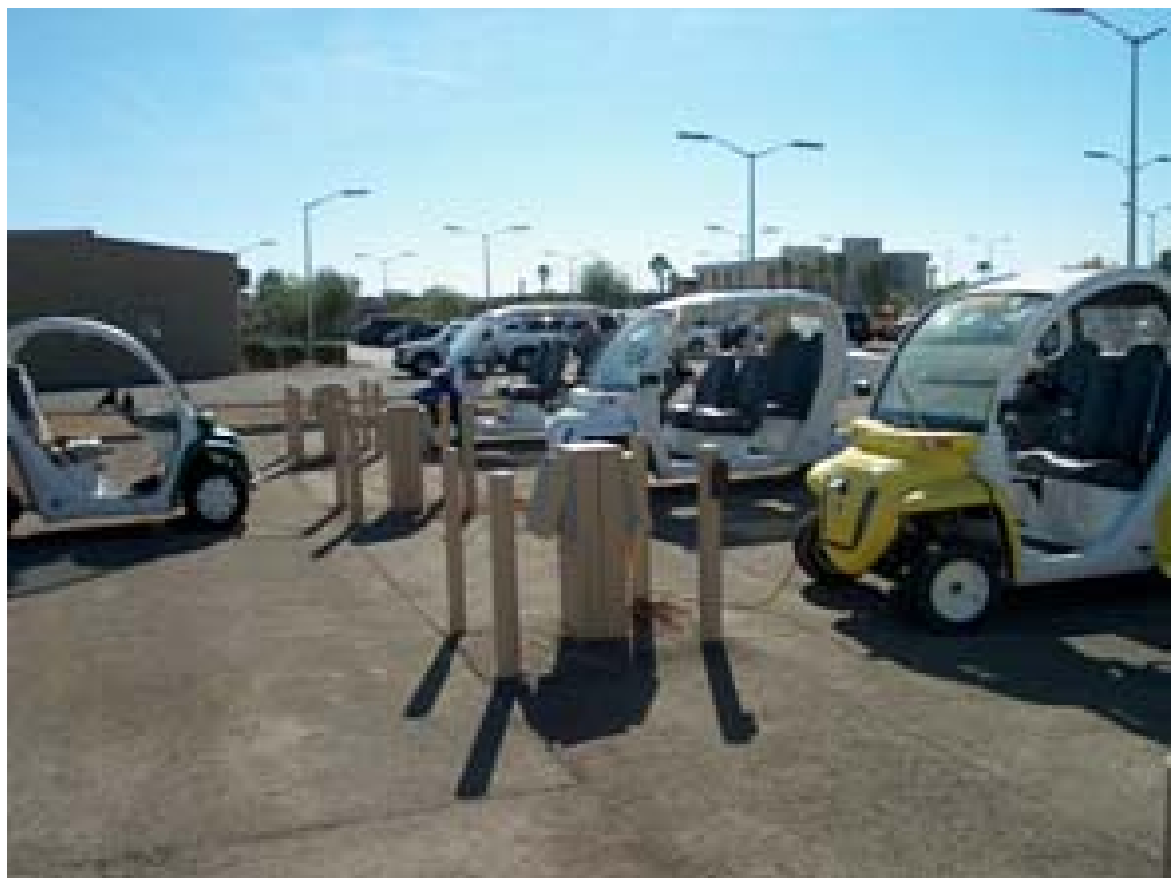

Figure 3. NEV parking lot charging at Luke Air Force Base.

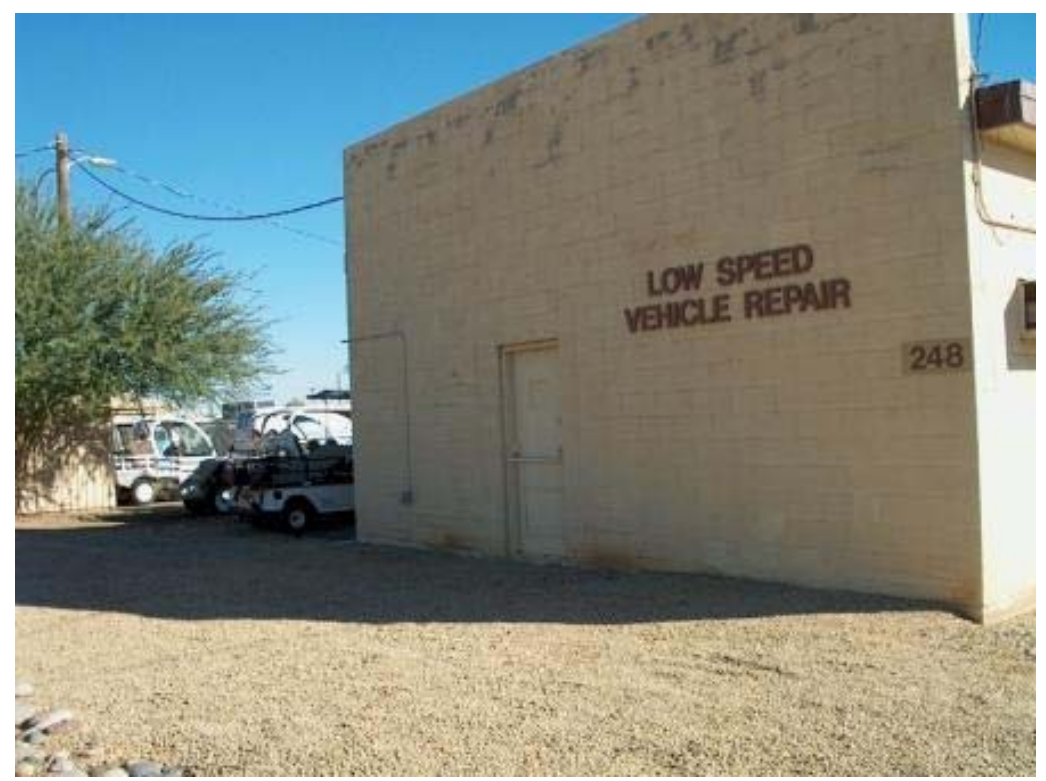

Figure 4. Luke Air Force Base vehicle repair shop.

\subsection{Marine Corps Recruit Depot San Diego}

One of two countrywide basic training bases used for training all new United States Marine Recruits is the Marine Corps Recruit Depot San Diego (MCRDSD), which is the location for all Marine Corp basic training on the west coast. The base, located in San Diego, California, encompasses 385 acres. The posted speed limit on the base is $20 \mathrm{mph}$, making this another ideal application for the implementation of a NEV fleet for speed-limited, short-range transportation. 
The MCRDSD NEV test fleet consisted of 10 GEM vehicles. One of the GEMs was used by the administration group, and the remaining nine GEMs were modified with a "police-package" consisting of code lights located on the windshield, a siren, horn, and specialized bumpers with towing capabilities. The Military Police Force used the modified police-package GEM NEVs for a variety of functions, including base patrol, speed and traffic enforcement, the towing and placement of traffic control signs and monitors, and the transportation of the canine units (in their carriers) to assigned work areas.

\subsection{City of Palm Springs Fleets}

The City of Palm Springs, California, self-proclaimed as the world's most famous desert playground, is a premier vacation destination and a growing metropolitan community. NEV fleet interest by the Palm Springs' city managers was enhanced by the opportunity for state funding to assist in purchasing NEVs, and the Palm Springs NEV program commenced in 2001. An $\$ 85,000$ grant from the California Energy Commission assisted with the purchase of 21 NEVs. With the addition of $10 \mathrm{NEVs}$ purchased with funding from other sources, there were a total of $31 \mathrm{NEVs}$ in fleet testing in the City of Palm Springs area.

The NEV fleet was established through a partnership between the City of Palm Springs and the Agua Caliente Band of Cahuilla Indians, the Palm Springs Unified School District, the Palm Springs Chamber of Commerce, and local private citizens. The goal of the Palm Springs NEV program was to demonstrate the potential for low-speed electric vehicles to substitute for gasoline-fueled vehicles, in a local municipality, while driving on designated (35 mph or less) roadways in the City of Palm Springs. The California Energy Commission asked the city to keep track of mileage and maintenance for a period of 1 year. Another objective of the program was to reduce vehicle emissions.

The City of Palm Springs further agreed to participate in the AVTA NEV data collection effort and to report their collected fleet data. To maximize NEV use, the city entered into a cooperative agreement with Electric Transportation Applications to use four LSV-100 "Fast Chargers" in four locations within Palm Springs. In addition to the regular NEV offboard chargers (Figure 5), two of the fast chargers were located at City Hall (Figure 6), the others were installed at the Parks and Recreation Building and the Palm Springs International Airport. Fast charge kits were installed on the NEVs to allow for rapid charging of high-use vehicles.

Twelve of the 31 GEM NEVs were allocated by the City of Palm Springs as follows; five for airport services, two for the Police Department, two for the City Yard and one vehicle each to the Chamber of Commerce, Information Services/Print Shop, and City Hall. Palm Springs Unified School District used three GEMs as part of an operating fleet.

Agua Caliente Spa Hotel and Casino in Palm Springs operated a fleet of six GEMs. Administrative, Building Maintenance, Computer IT, and Security staff used these vehicles.

An innovative businessman initiated a rental business, GEM Rentals of Palm Springs, located in the downtown area. The 5-vehicle fleet consisted of 2- and 4-passenger GEMs. The rental vehicles were used by tourists as a fun and colorful alternative form of transportation for recreational and sightseeing use. Although the concept was unique and applicable to the city, it did not result in a viable long-term business, and the vehicles were sold.

NEV use by five private citizens was monitored and included in the private citizen fleet data. These vehicles were used for a variety of purposes; one was used to assist with duties as a crossing guard at the school, another private citizen used the vehicle for use in a small pool maintenance and service business, and the remaining three NEVs were used for personal transportation. 
All the NEVs in the Palm Springs fleet were manufactured by GEM. A total of 42,247 miles were accumulated on the 31 NEVs.

\subsection{Palm Valley Private Parties}

Another private fleet included in the AVTA NEV data collection effort was in Palm Valley, Arizona, a suburb located 18 miles west of downtown Phoenix. Six of the GEM NEVs were converted to be able to use a fast charger, which was installed at the community golf club (Figure 7), and it was accessible to the public. These vehicles were typically used for personal transportation in the Palm Valley community and for golf. The same dealer supporting the LAFB fleet vehicles provided maintenance to the Palm Valley vehicles. Overnight charge infrastructure was available at each NEV owner's home.

In the government fleets, the fleet managers were allowed to develop the infrastructure necessary to support NEV operation, including charging, maintenance, mission selection, and operator training, and a fast charger was provided to the fleet manager to assist with charge infrastructure development. For private vehicles operated in Palm Valley and Palm Springs, a fast charger was installed at a location convenient to the NEV owners.

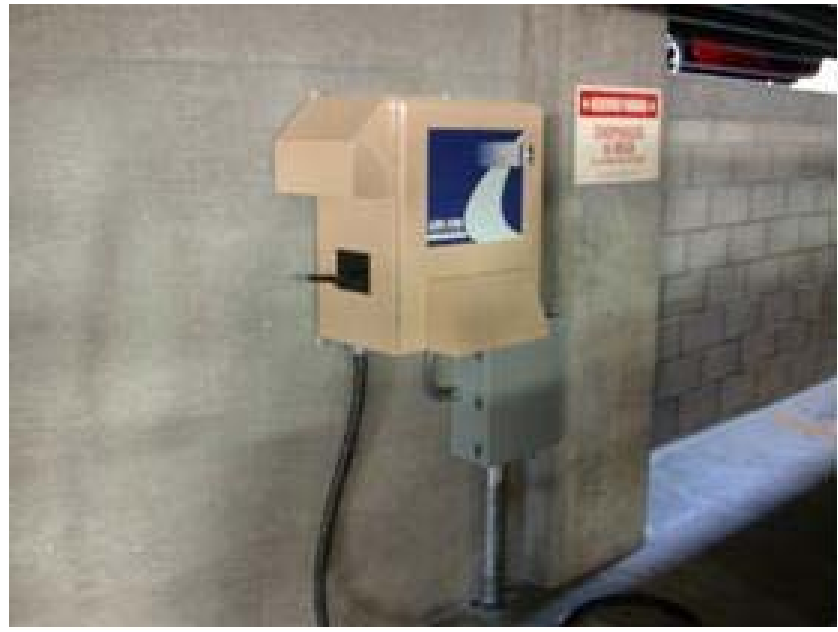

Figure 5. LSV-100 NEV charger in the City of Palm Springs' City Hall parking garage.

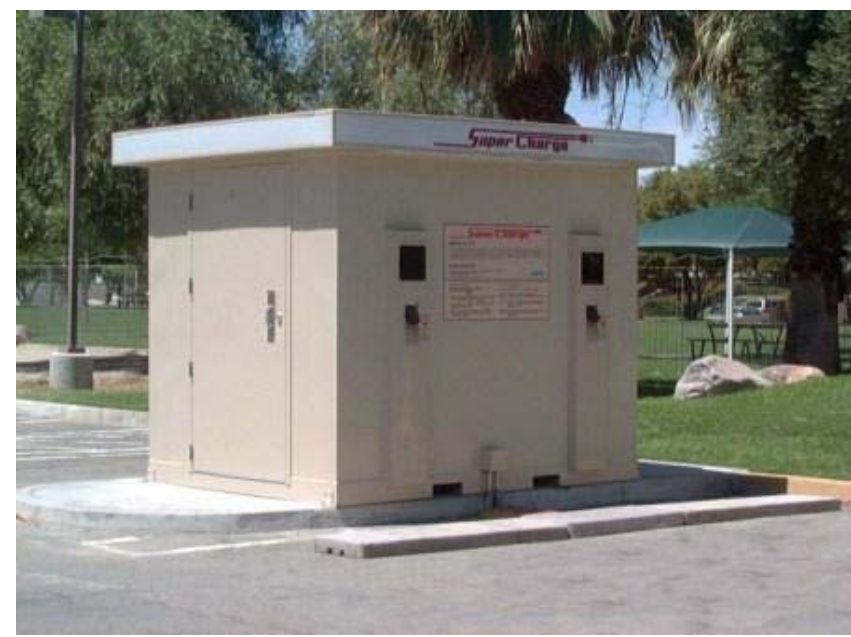

Figure 6. City of Palm Springs SuperCharge Station. 


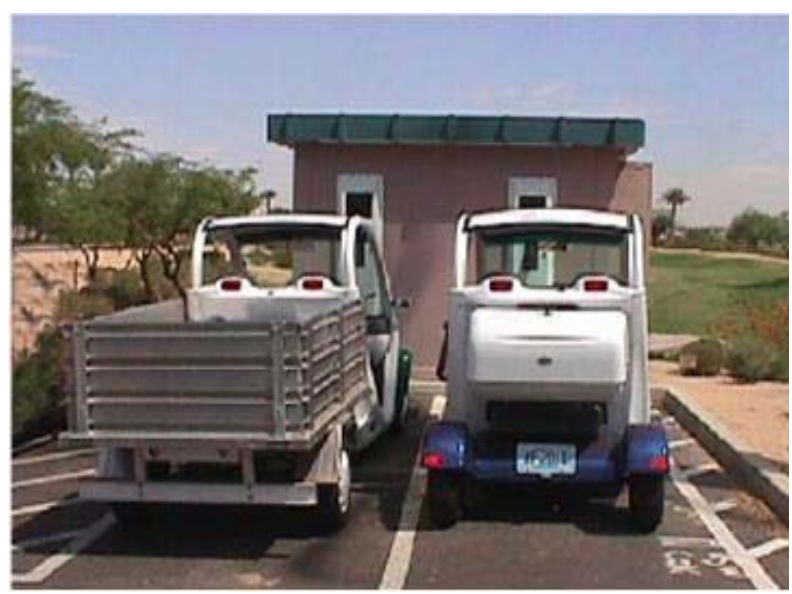

Figure 7. Palm Valley SuperCharge Station.

\subsection{Fleet Testing Results}

A total of 168,419 miles (Table 2) were accumulated on the 101 NEVs. Various types of GEM models were included in the fleet. These included 2-passenger, 4-passenger, and long-bed and short-bed models. Some vehicles were modified with option packages to better accommodate functional use.

Although no formal mission criteria were identified to purchasers of NEVs participating in the testing, misapplication of vehicles was rarely a problem. Vehicles were typically applied into missions in which a daily reliable single-charge range was sufficient to support the mission. In a few circumstances, vehicles were placed in private applications into missions where daily range requirements were beyond the reliable single-charge performance of the vehicle. For example, a swimming pool service in Palm Springs attempted to use a GEM for conducting its service route. Although a serious effort was made to adjust the route and opportunity charge using the Palm Springs fast charger, the effort was abandoned as the fast charger was not convenient to the pool service route. Rather than provide range extension, the fast chargers were used to replace overnight charge infrastructure. The fast charger could rapidly recover NEVs that were unable to charge overnight because of limited convenience outlet availability or because the NEV was not being plugged in.

Charge infrastructure was a serious problem at LAFB and, to a much lesser extent, in all other fleets. As NEVs charge from a Level 1 ( $120 \mathrm{~V}$ single phase) convenience outlet available in every electrified building, charging infrastructure was typically not an issue in the minds of NEV purchasers. When vehicles were aggregated in small numbers, sufficient convenience outlets were typically available to support charging. However, when large numbers of vehicles were aggregated, such as at LAFB, the available Level I $120 \mathrm{~V}$ convenience outlets were not sufficient to support charging needs. This resulted in overloading circuits by connecting multiple vehicles to a single circuit, vehicles not being charged, and extension cords stretching throughout NEV parking locations.

Vehicle maintenance was an issue in all fleets, becoming more critical in fleets such as LAFB where the NEVs were heavily used. Maintenance issues were both economic and logistic. Many NEV users had not anticipated the maintenance requirements of their vehicle and had no budget to maintain the vehicle. This resulted in vehicles being out of service for extended periods (often months) while waiting for repairs to be funded. When maintenance could be funded, it was necessary to use a GEM dealer or GEM mobile service (when available). Both sources required advanced scheduling and travel (either by the technician or the vehicle), which required several days to weeks to organize. Travel requirements also significantly impacted maintenance costs. Examples of maintenance costs for selected repairs are shown in Table 3. 
Table 2. NEV fleet mileage accumulated.

\begin{tabular}{lcr}
\hline NEV Fleet & Number NEVs & Total Miles \\
\hline United States Air Force_Luke Air Force Base & 55 & 113,317 \\
United States Marines_-MCRDSD & 10 & 11,858 \\
City of Palm Springs_City Fleet & 12 & 14,990 \\
Palm Springs_-Agua Caliente Band of Cahuilla Indians & 6 & 11,349 \\
City of Palm Springs_-Public School District & 3 & 4,915 \\
Palm Springs_-Private Vehicles & 5 & 5,717 \\
Palm Springs_-GEM Rentals & 5 & 5,276 \\
Palm Valley_Private Vehicles & 5 & 997 \\
\hline Totals & 101 & 168,419 \\
\hline
\end{tabular}

Table 3. Sample NEV fleet testing maintenance costs.

\begin{tabular}{lllc}
\multicolumn{1}{c}{ Vehicle Fleet } & \multicolumn{1}{c}{ Vehicle Type } & \multicolumn{1}{c}{ Maintenance Required } & Maintenance Cost \\
\hline \multirow{2}{*}{ LAFB } & GEM Long Bed & Battery Replacement & $\$ 1,035$ \\
& & Charger Replacement & $\$ 1,015$ \\
& & Controller Replacement & $\$ 565$ \\
& & Tire Replacement & $\$ 465$ \\
\hline City of Phoenix & \multirow{2}{*}{ GEM 4 Passenger } & DC-DC Converter Replacement & $\$ 665$ \\
\hline
\end{tabular}

Unfortunately, significant amounts of maintenance were required in every NEV fleet. Maintenance can be categorized as either preventative (routine service to maintain the vehicle) or corrective (repair required to correct a vehicle breakdown).

\subsubsection{Preventative Maintenance}

By far, the largest amount of preventative maintenance (and the most troublesome) involved watering batteries in NEVs equipped with flooded lead-acid (FLA) batteries. Virtually all NEVs in the AVTA fleet testing were equipped with FLA batteries. However, six vehicles at LAFB were retrofitted as part of a test with valve regulated lead-acid (VRLA) batteries; VRLA are sealed and require no watering.

The following issues were associated with the watering of FLA batteries:

- $\quad$ Battery watering is a responsibility of the vehicle owner/operator.

- Many fleets contained only administrative personnel who were not trained or equipped to perform vehicle maintenance.

- $\quad$ To be performed properly, battery watering requires personal protective equipment for the person conducting the watering and a proper facility for neutralizing any acid that may drip from the batteries during or after watering when the batteries are charged.

- $\quad$ Battery watering was very often not performed as no one was directly responsible to maintain the NEVs and/or no one was properly trained and equipped to do so. 
- $\quad$ Many FLA batteries failed prematurely due to not being watered and subsequently drying out.

Unlike a golf cart, a GEM (the most popular NEV) has a significant parasitic load on the battery even when the vehicle is turned off. This load can discharge the vehicle battery in less than 2 weeks. Therefore, a disconnect switch is provided under the driver's seat to completely disconnect the battery if the vehicle is to be inoperable for more than a few days. Preventative maintenance is required to turn disconnect switches to the "off" position or to place the NEVs on charge as necessary to prevent battery discharge. If this is not done properly, the vehicle battery is discharged to near zero state-of-charge (SOC). At this low level of charge, the onboard vehicle charger will not charge the battery, and the vehicle must receive corrective maintenance (repair) to charge the battery. In addition, discharge to this SOC level often results in battery failure. The inability to maintain a proper level of battery charge resulted in extended out-of-service periods and premature battery failure for vehicles in all the test fleets.

Very few vehicles were driven sufficiently to require high-mileage-related preventative maintenance such as tire and brake replacements. The tire replacement that occurred was typically required because of sidewall cracking, resulting from exposure to strong solar radiation in the Southwest United States.

\subsubsection{Corrective Maintenance}

The majority of corrective maintenance was battery related. The following corrective maintenance was very typical in all fleets:

- $\quad$ Low vehicle range requiring battery replacement. Batteries often failed prematurely (1 year or less) due to drying out from lack of watering, overdischarge due to vehicle inactivity, or overdischarge due to charger failure. Properly maintained batteries typically provided a 3-year service life.

- $\quad$ Charger failure. Onboard charger failure in GEM NEVs was common in all fleets. The problem was particularly acute for vehicles that were operated frequently and charged for extended periods every day. Chargers through model year 2005 were not sealed and many failed because of exposure to the environment.

- $\quad$ Controller failure. Motor controller failure in GEM NEVs was common in all fleets. No predominate reason for these failures was evident from fleet testing data.

- $\quad$ Motor failure. Motor failure in GEM NEVs was clustered in missions that required high power (hill climbing or carrying heavy loads). These failures were typically associated with motor overheating.

- $\quad$ DC-DC converter failures. DC-DC converter failure was common in all fleets. The converter is not sealed and is placed in a location that is subject to water splash. Corrosion of the converter printed circuit board and fuse connections was normally the cause of converter failure.

Availability of repair parts, qualified technicians, and maintenance facilities was frequently an issue. Clearly the lack of onsite maintenance for large fleets, such as LAFB, was a significant detriment to successful vehicle operation.

\subsection{NEV Fleet Testing Lessons Learned}

For NEVs to be successful, the vehicle must be viewed by the user as a tool and not a toy. AVTA NEV fleet testing provided significant insight into the issues facing managers in establishing effective 
operations and the adoption of NEVs, as tools, in fleet applications. Lessons learned from the fleet testing are presented below and are discussed in more detail in the applicable sections of this report.

\subsubsection{Mission Selection}

- $\quad$ Mission selection can be done informally when the total daily operating range requirement is within the single-charge capability of the NEV.

- Mission selection must be more formally conducted when opportunity charging is required to extend vehicle range beyond single-charge capability. Mission requirements must be coordinated with charging infrastructure and vehicle selection.

\subsubsection{Charge Infrastructure}

- Charge infrastructure is not an issue for vehicles owned and operated by individuals out of their home. Convenience outlets are available for charging and missions typically do not require opportunity fast charging.

- $\quad$ Charge infrastructure for fleet vehicles must be formally provided. Where multiple vehicles congregate, convenience outlets provided for normal building services are not sufficient to provide charging power. Additional charging outlets must be provided, preferably in close proximity to NEV parking locations.

- Fast charging can provide a backup to overnight charging when vehicles, for whatever reason, do not receive an overnight charge.

- $\quad$ Fast charging is not routinely used unless vehicle missions require opportunity fast charging.

\subsubsection{Maintenance}

- $\quad$ Preventative and corrective maintenance must be available promptly and at a reasonable cost to support NEV operations. Fleets of even moderate sizes should consider establishing contract or inhouse maintenance capabilities to support their NEV operations.

- $\quad$ Preventative maintenance (including battery watering) should be the function of contract or in-house maintenance staff and not a function of the operator.

\subsubsection{Operator Training}

- $\quad$ Operators in all fleets must be made aware of vehicle range limitations, unique requirements (such as disconnect switches), and preventative maintenance requirements (such as battery watering). 


\section{DETERMINATION OF NEV MISSION}

\subsection{Daily Mission}

Understanding the daily mission requirements of NEVs in fleets is critical to ensuring successful deployment. A potential mistake when first deploying NEVs into fleets is putting them into applications where the NEV performance doesn't meet the mission requirements. The following suggestions are provided to assist with estimating range requirements.

\subsubsection{Record Daily Routine Vehicle Miles}

Range is often the most critical area to understand in existing internal combustion engine fleets. Recording daily mileage on candidate replacement fleet vehicles for a period long enough to represent that vehicle's typical daily driving pattern can provide valuable information in selecting the appropriate vehicle and setting up the necessary infrastructure. A range of more than 30 miles per day will likely require fast charging as part of the overall fleet design because using the onboard charger at $120 \mathrm{~V}$ is typically an 8 to 12 hour charge process.

\subsubsection{Note Extraordinary Miles}

If there are occasions when longer trips occur outside the normal routine and outside of the capabilities of the NEV, it may be necessary to have a backup vehicle available.

\subsubsection{Identify Daily Route Stops}

Opportunity charging during the day can extend the range by up to 5 times the original range when fast charging is used. Even using the $120 \mathrm{~V}$ onboard slow charger can provide additional range extension if the NEV is parked at a charging location for 1 or more hours. Fleet drivers should document where they stop within their typical daily routes and approximately how long they are at that particular location. Locations where NEVs are parked for at least 20 minutes are ideal candidates for a fast charger. Locations where the vehicle is parked for 2 hours or more are candidates for plugging into a convenience outlet to provide more limited range extension.

As a general rule of thumb, the following range extension can be expected:

- $\quad 120 \mathrm{~V}$ Onboard Charger: 0.1 mile per minute of charging

- $\quad$ Offboard Level 3 Fast Charger: 1.0 mile per minute of charging.

\subsection{Payload Requirements (Passenger or Cargo Haulers)}

Typical payloads for passenger NEVs range from 500 to 800 pounds, and NEV cargo haulers range from 600 to 1,000 pounds. Examples of one manufacturer's available payload (Payload = Gross Vehicle Weight - Curb Weight) for passenger and cargo hauler NEVs are shown in Figures 8 and 9, respectively. 

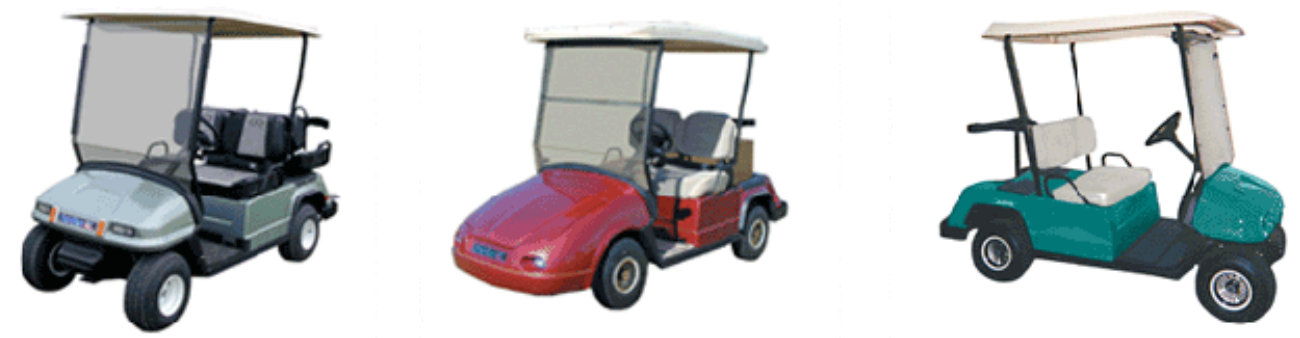

Figure 8. Payload for the Columbia ParCar Passenger NEV is 750 pounds.

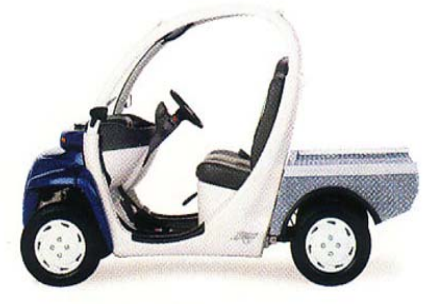

GEM 2 Passenger Short-back NEV

CURB WEIGHT-1,160 lb GVW-1,850 lb

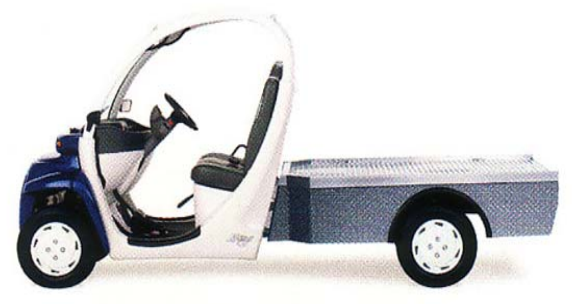

GEM 2 Passenger Long-back NEV

CURB WEIGHT-1,240 lb

GVW-2,300 lb

Figure 9. Typical payloads for NEV cargo haulers are up to 1,000 pounds.

\subsection{Vehicle Options for Extreme Climates}

Most NEV manufacturers include a long list of options for their vehicles that include heaters, doors (both hard and soft canvas), defrosters, etc. It's important to consider that the options requiring electricity provided from the vehicle battery will negatively impact driving range. Fleets located in cold climates should plan to garage the vehicle in a heated area for charging and should consider fast charging to boost the daily range of the NEVs.

\subsection{NEV Limitations}

It's important for the user to understand the limitations of NEVs and assign the vehicles to applications within those limitations. Aggressive salesmen could overstate the capabilities of their vehicles in order to achieve a sale, but the results will likely end in the NEV assigned to an application that does not meet its capabilities and ultimately lead to dissatisfaction with the product.

If the NEV manufacturer has not participated in the NEVAmerica testing, the prospective NEV fleet manager should consider calling on actual NEV users that operate that NEV under consideration in order to obtain neutral real-world performance feedback. 


\section{NEV SELECTION}

\subsection{Configurations}

As shown in Figures 8 and 9, there are various vehicle configurations available to meet fleet needs. Most NEVs are open-air design and have optional soft or hard doors. Figure 10 shows a NEV that is fully enclosed with roll-up windows.

\subsection{Batteries}

As mentioned earlier, there are generally two types of batteries found in NEVs. The most common is a deep-cycle FLA battery while some NEV manufacturers also use a sealed VRLA battery. The characteristics of each type are listed in Table 4. From a practical standpoint, the fleet manager should take many things into consideration in addition to the battery performance and cost. Specifically, the capability to consistently water FLA batteries must be considered. Without such watering capability, VRLA batteries are a more appropriate choice.

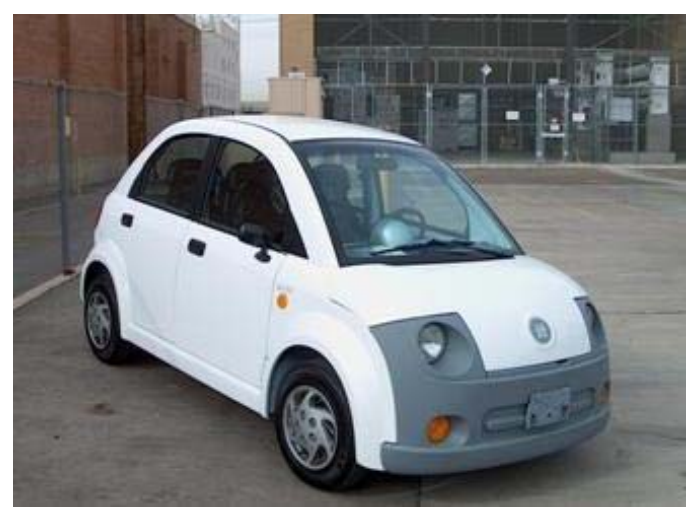

Figure 10. Dynasty "It".

Table 4. Lead-acid battery type comparisons.

\begin{tabular}{|c|c|c|}
\hline & Flooded (FLA) & GEL (VRLA) \\
\hline Average Retail Cost & $\$ 60$ per $\mathrm{kWh}$ & $\$ 120-180$ per $\mathrm{kWh}$ \\
\hline Total $\mathrm{kWh}$ per vehicle & $7.2 \mathrm{kWh}$ & $7.2 \mathrm{kWh}$ \\
\hline Energy Density $(\mathrm{Wh} / \mathrm{kg})^{\mathrm{a}}$ & $15-26$ & $22-33$ \\
\hline Power Density (W/kg) & $50-100$ & $100-137$ \\
\hline Estimated Cycle Life $^{\mathrm{b}}$ & Up to 600 & $150-300$ \\
\hline $\begin{array}{l}\text { Charge Retention ( } 50 \% \\
\left.\text { capacity, } 27^{\circ} . \mathrm{C}\right)^{\mathrm{a}}\end{array}$ & 90 Days & 1.5 Years \\
\hline Fast Charging & Yes & Yes \\
\hline Charging Locations & $\begin{array}{l}\text { Outdoor Only or proper ventilation to meet code } \\
\text { requirements (significant gassing during charge) }\end{array}$ & Indoor or Outdoor \\
\hline Maintenance & Monthly watering and cleaning ${ }^{\mathrm{c}}$ & Occasional visual inspection \\
\hline $\begin{array}{l}\text { a. Crompton, T.R; Battery } \\
\text { b. Assumes proper mainte } \\
\text { c. Actual watering frequen }\end{array}$ & $\begin{array}{l}\text { ference Book, Second Edition; Table } 2.11 \\
\text { ce, charging, and discharge control. } \\
\text { depends on use; the user may need to adjust appr }\end{array}$ & \\
\hline
\end{tabular}




\subsubsection{Battery Cost}

Battery costs vary greatly by manufacturer and battery technology. For a typical $72 \mathrm{~V}$ NEV battery pack, you can expect to pay from $\$ 600$ for six $12 \mathrm{~V}$ FLA batteries to $\$ 1,000$ for six $12 \mathrm{~V}$ VRLA batteries. The premium cost of gel VRLA batteries must be balanced against the benefits they provide such as minimal maintenance requirements. In addition, VRLA batteries are not treated as hazardous materials for storage and shipping, and they can be charged indoors without the special ventilation required for FLA batteries.

\subsubsection{Energy Density}

Energy density is the measure of how much energy is available from the battery for its weight $(\mathrm{Wh} / \mathrm{kg})$ and varies based on the discharge rate. For a specific discharge rate the energy density is sometimes referred to as the "Specific Energy."

\subsubsection{Power Density}

Power density measures the ability of the battery to provide its stored energy to produce usable work $(\mathrm{W} / \mathrm{kg})$. This characteristic is important for applications that have high discharge rates. These applications include hill climbing and moving large loads. Applications of this type can be performed more consistently with a battery providing high power density. Generally, VRLA batteries have significantly greater power densities than FLA batteries.

\subsubsection{Estimated Cycle Life}

Actual cycle life is the most difficult battery characteristic to determine. Many factors, both related to the battery and to the application the battery is used in, impact the number of cycles that a battery will last at a given performance level. Often, batteries are put in improper applications from which they were originally designed and the battery takes the blame. For example, batteries that were designed for low discharge rate would not perform well in an application that requires a high discharge rate. Unfortunately, this is seen all the time in all kinds of applications. The typical result is a battery that underperforms by not having the voltage stability required to operate the vehicle at peak performance. In extreme cases, cycle life can be considerably negatively impacted as well.

Other aspects that impact battery life include:

- Improper maintenance — For flooded batteries that require regular watering, not performing watering as recommended by the battery manufacturer will significantly impact battery life.

- Improper charging - Improper charging can significantly impact the cycle life of a battery. This is especially true for VRLA batteries that are intolerant of overcharge. A common mistake is to install VRLA batteries in place of FLA batteries without modifying the charger. The FLA battery charger subsequently overcharges the VRLA batteries reducing their cycle life.

- $\quad$ Overdischarge - Overdischarge events significantly reduce battery life. VRLA batteries are particularly sensitive to overdischarge. When NEVs are selected, a key factor should be the vehicle's ability to control overdischarge from extended vehicle operation and to function after long periods off charge. 


\subsubsection{Charge Retention}

Charge retention describes the number of days that it takes for the battery to self-discharge to a specified level SOC. Table 4 specifies the number of days to discharge to $50 \%$ of capacity at $27^{\circ} \mathrm{C}$. This assumes no other parasitic loads on the battery pack, such as DC-DC converters or other accessories that many NEVs may keep energized, even though the vehicle is turned "off." Charge retention is a strong advantage of VRLA batteries as they have very low self-discharge rates. This means VRLA batteries have a longer shelf life than FLA batteries. It is important, however, that parasitic loads on the battery be controlled or accommodated. Often, these loads are many times greater than the batteries self-discharge rate and will fully discharge the battery in a matter of 7 to 10 days if it is not charged or disconnected from the parasitic load. Ironically, while VRLA batteries have a lower self-discharge rate, they are less tolerant of overdischarge caused by failure to control parasitic loads.

\subsubsection{Fast Charging}

Fast charging NEVs typically refer to charging them from approximately $20 \%$ SOC to approximately $80 \%$ SOC in less than 1 hour. Both FLA and VRLA batteries can be fast charged. Fast charging requires a smarter charge system than a traditional slow charger in order to avoid destructive overcharge and to provide adequate safety measures. At a minimum, the fast charger should be temperature compensated, should have a pilot connection for rapid shutdown in the event the charge connector is pulled out (disconnected) while the battery is under charge, and it should have a UL or equivalent listing.

\subsubsection{Charging Location}

During charge, an FLA battery may have considerable gassing. It is important, therefore, to charge in an open area, or a facility that meets National Electric Code ventilation requirements for FLA batteries. ${ }^{d}$ VRLA batteries are considered nonvented storage batteries and are exempt from such ventilation requirements. ${ }^{\mathrm{e}}$

\subsubsection{Maintenance}

FLA batteries require regular maintenance, including watering and cleaning of the batteries, the battery terminals and the battery box to ensure that acid deposits are neutralized before corrosion damage occurs. Original equipment manufacturer (OEM) maintenance guidelines should be followed. VRLA batteries do not require regular maintenance, but it is wise to visually inspect the batteries every quarter to ensure terminals are still properly fastened and that there are no visible abnormal conditions.

\subsection{Chargers}

NEVs are typically equipped with an onboard overnight charger that can be plugged into a $120 \mathrm{~V} /$ 15 amperes (amps) convenience outlet. Charge times for the onboard charger are typically 8 to 12 hours. ${ }^{f}$ Onboard chargers are normally programmed specifically for the battery type that was installed at the factory (FLA or VRLA). Charger recalibration or replacement is imperative if the battery type is changed.

\footnotetext{
${ }^{\mathrm{d}}$ NFPA 70; Article 625-29c

${ }^{\mathrm{e}}$ NFPA 70; Article 625-29d

${ }^{\mathrm{f}}$ NEV America Data 2002 Data Sheets
} 
Optional offboard opportunity fast chargers are available that provide added operational flexibility. Modifications are necessary to the NEV to accommodate connection to the opportunity fast charger; aftermarket kits are available. In some cases, the vehicle OEM can make the modifications at the factory or have an authorized up-fitter make the modifications prior to delivery of the NEV to the customer location.

Additional information on chargers can be found in the Infrastructure Section of this report.

\subsection{Accessories}

There is an abundance of accessories for NEVs offered by OEMs and by aftermarket companies. Accessories may include:

- $\quad$ Bumpers

- $\quad 12 \mathrm{~V}$ accessory plug

- $\quad$ Hard or soft doors

- $\quad$ Security lighting and sirens

- Heater/defroster

- Cargo container options

- $\quad$ Optional batteries

- $\quad$ Emergency lights.

In addition, there are several NEV up-fitters that can provide custom vehicle configurations such as security vehicles.

\subsection{Warranty \& Service Support}

Warranty and service support varies by manufacturer and should be carefully reviewed prior to purchasing a NEV. Service requests can be handled through onsite service calls or by the local service facility picking-up a NEV and returning it when the service is complete.

Issues to be examined include what parts are stocked locally for NEV repair, what are typical repair times, and what is a typical repair cost (including travel time or towing as applicable). If a large number of NEVs are aggregated at one location, contract maintenance may provide a more cost effective alternative than dealer-based maintenance. The NEV dealer should be consulted before the vehicle purchase to confirm that contract maintenance will not void warranty terms.

\subsection{Parts Cost and Availability}

The cost and availability of replacement parts outside of warranty should be compared between the NEV manufacturers. The NEV mission and the number of miles driven can impact the life of various components. Adequate budget reserves should be put in place to cover miscellaneous items that may need replacing beyond the warranty period. NEV replacement items can include: 
- $\quad$ Battery packs

- $\quad$ Electronic cards

- $\quad$ Motor controllers

- $\quad 120 \mathrm{~V}$ onboard chargers

- $\quad$ Tires.

\subsection{Specifications and Performance}

Data sheets summarizing the results of the AVTA's NEVAmerica baseline performance testing program are a good source of information concerning the performance of a particular NEV model. Figure 11 shows an example of a NEVAmerica data sheet.

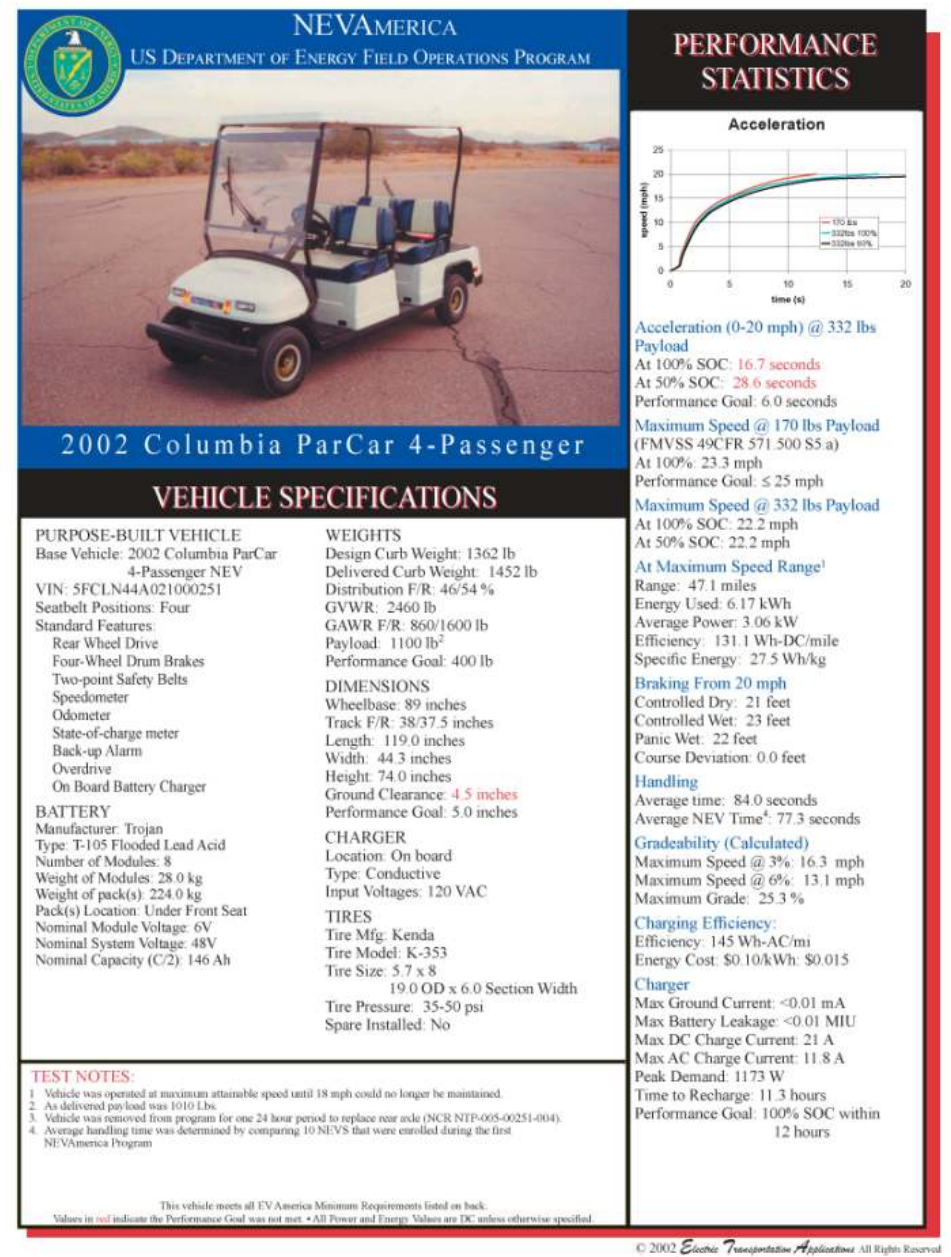

Figure 11. Example NEVAmerica data sheet. 
Specifications are detailed on each data sheet. Every NEV that has a published NEVAmerica data sheet has met the minimum NEVAmerica requirements as detailed on the back of the data sheet. Prior to the actual NEVAmerica testing, input on performance goals was provided by OEMs, fleet operators, and other industry stakeholders. These performance goals are listed on the front of the data sheet. Those goals that are not met by the particular NEV tested are shown in red. All NEVAmerica data sheets are attached in Appendix 3. Additional information can be found at the AVTA testing results Website (http://avt.inl.gov).

Other resources available to potential NEV users include actual fleet managers that currently operate NEVs. Sales representatives should readily provide references of other fleets operating NEVs.

\subsection{Vehicle Documentation}

The availability and completeness of vehicle documentation is an important evaluation criterion. This is especially important if maintenance will be performed in-house. Manuals that should be available include:

- $\quad$ Operators manual

- $\quad$ Troubleshooting guide

- $\quad$ Maintenance manual

- $\quad$ Recommended spare parts list

- $\quad$ Complete parts price catalog.

Review the available manuals for completeness and discuss their accuracy with fleet managers that are currently operating and maintaining a NEV fleet.

\subsection{Battery Transporting/Storage}

There are certain restrictions and requirements on the transporting and storage of FLA batteries. These batteries are considered hazardous material and must be handled as such when transporting and storing onsite. This includes certain restrictions on the number of batteries you can maintain onsite without a special permit and special shipping requirements. VRLA batteries are not considered hazardous material and, therefore, can be transported and stored onsite with minimal requirements. ${ }^{\mathrm{g}}$

\subsection{Battery Recycling/Disposal Considerations}

The NEV OEM should provide guidelines on recycling and/or disposing of the batteries that came with the NEV. If you purchase batteries from a battery supplier other than the OEM, they also should provide similar guidelines. It is the owner's responsibility to properly dispose of used batteries. In most cases, this procedure is no different than disposing of starting batteries from internal combustion engine vehicles.

g 49 CFR parts $171-180$ 


\section{INFRASTRUCTURE}

\subsection{Charging/Electrical Infrastructure Requirements}

One of the typically most overlooked areas when purchasing NEVs for fleet applications is the charging infrastructure. For consumer use, the NEV charging infrastructure is very straightforward. Charging is typically done using the onboard charger and the $120 \mathrm{~V}$ outlet in a garage or carport. This infrastructure meets the needs of a user who is not a high-mileage user. Fleet users very quickly run into situations where there is nowhere to plug in their vehicles. Even though there may be multiple outlets available, typically they share the same circuit breaker and will overload and trip it if more than one vehicle is connected. Charging infrastructure for fleet applications of NEVs must, therefore, be carefully planned prior to receiving NEVs.

Only $50-70 \%$ of the nominal vehicle range should be used. This allows a margin to accommodate circumstances when the NEV is operated in cold weather, is operating with an older battery having lost some capacity, or is operating under more demanding conditions than originally estimated. It is imperative that the NEV reliably fulfill the mission it is assigned. If it even infrequently is unable to complete its mission, the vehicle will not be viewed as a useful tool. In circumstances where more than $50 \%$ of nominal vehicle range is to be used, opportunity charging infrastructure should be provided to ensure that a "safety valve" is available to overcome circumstances in which the vehicle may be range limited.

Regardless of the vehicle's range requirements, provisions should be made to allow every vehicle to plug in its onboard charger each night for charging. A typical overnight charge will take 8 to 12 hours and will return the battery to $100 \%$ SOC. Power requirements for onboard chargers are $120 \mathrm{VAC}$ single phase, with a maximum current draw of 12 amps. This requires a dedicated 15 amp supply circuit for each NEV. Charging power should be located as close as possible to where the vehicle is parked overnight to make charging as convenient as possible.

Opportunity charging can be provided in limited circumstances by the onboard charger. A typical onboard charger will deliver approximately 0.1 mile per minute of charge. However, field experience has shown that significant use of the onboard charger for opportunity charging can result in its premature failure.

Where significant opportunity charging is required, an offboard fast charger is required.

Experience with fast charging at LAFB has shown that the fast charger can deliver approximately 1.0 mile of range extension, per minute of charge, at a charge rate of 100 amps. An example of an opportunity fast charger available on the market today is the LSV-100 EV Opportunity Charger from Electric Transportation Engineering Corporation (eTec), which is shown in Figure 12. The LSV-100 provides a maximum direct current output of $100 \mathrm{amps}$ and can automatically identify common voltage battery packs including $36,48,72$, and $96 \mathrm{~V}$. The LSV-100 requires a power supply of $208 \mathrm{VAC}$, threephase power at 40 amps maximum draw (50 amp breaker). A detailed specification of the LSV-100 is provided in Appendix 4.

Because the opportunity fast charger can charge the NEV in minutes instead of hours, locations where the user stops even for a few minutes can be ideal places to locate a fast charger. Placing the opportunity charger by such facilities as employee break rooms or lunch areas works well, often providing enough time to return the battery pack to an $80 \%$ or higher SOC. 


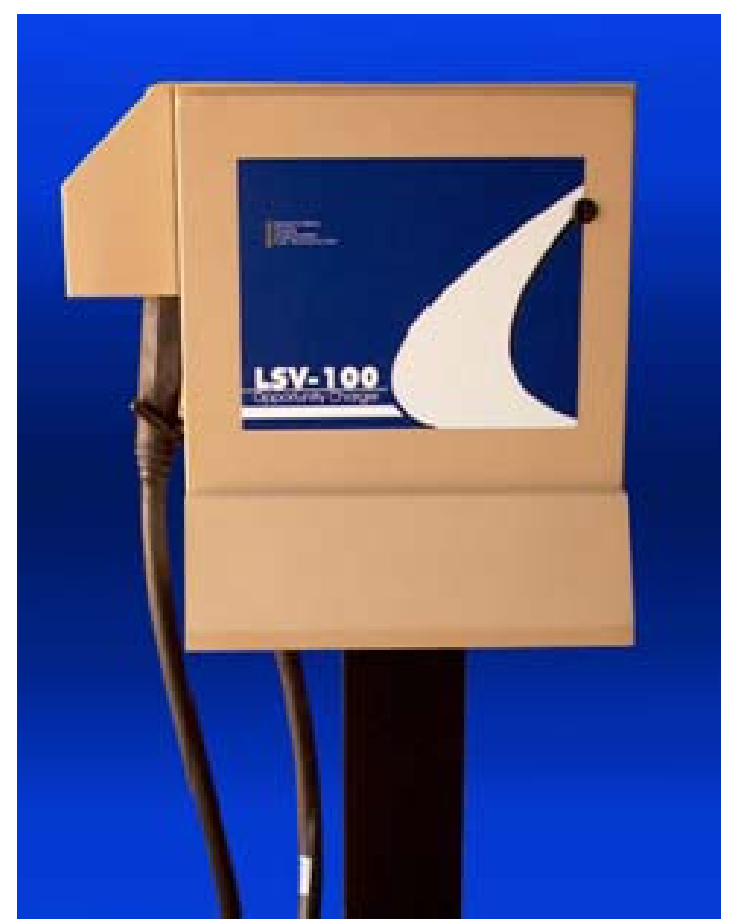

Figure 12. LSV-100 EV opportunity charger.

\subsection{Maintenance Infrastructure Requirements}

Maintenance infrastructure requirements will vary with the level of maintenance that is performed in-house. Unless maintenance facilities and mechanics are available onsite, contracting out all the preventative and corrective maintenance may be the best approach. However, if NEV maintenance will be performed in-house, the below issues must be addressed.

\subsubsection{Facility Requirements}

A standard garage is adequate for maintenance of NEVs. Charging power must be available, and a fast charger should be available to allow rapid recovery of vehicles prior to service. Adequate air exchange must be provided in cases where FLA batteries are charged and serviced. A vehicle lift hoist is not necessary as long as there are proper hydraulic jacks available.

\subsubsection{Special Tools}

All hand tools that are used in and around the battery packs need to be insulated in such a manner that they cannot arc across the battery packs or other source of voltage on the vehicle. This generally includes all wrenches, screwdrivers, and ratchets.

A digital multimeter that provides the basic function of measuring voltage and impedance is required for basic maintenance of the battery pack and troubleshooting the electrical system. An optional amp-probe may also be useful in certain diagnostic situations.

The NEV manufacturer should specify other special tools that are required to perform in-house maintenance. It may be useful to review the requirements with the Fleet Service Manager of the NEV 
manufacturer and also to speak with other fleet manager's that are currently providing their own in-house maintenance.

\subsubsection{Safety Equipment}

When servicing FLA batteries, personal protective equipment in the form of splash proof goggles or face shields, rubber gloves, and aprons must be provided and used. Personnel servicing batteries must be trained in the proper use of personal protective equipment to protect them from acid spills and splashes. In addition, proper facilities must be provided to neutralize battery spills and wash water.

\subsubsection{Spare Parts}

The NEV manufacturer should provide a list of recommended spare parts that includes a description of the part, part number, cost, and sources for purchasing parts (if not available through the manufacturer), and availability. Generally, items that should be considered for onsite stock (depending on the size of the NEV fleet) include the following parts:

\section{- $\quad$ Tires}

- $\quad$ Brake pads, assembly parts

- $\quad$ Spare battery modules

- $\quad$ Motor controller

- $\quad$ Motor maintenance parts (brushes, filters, etc.)

- $\quad$ Body panel fasteners

- $\quad$ Vehicle safety items

- $\quad$ Side view mirrors

- $\quad$ Seatbelts

- Replacement bulbs.

- Vehicle maintenance.

Proper vehicle maintenance documentation is critical to implementing a successful in-house maintenance program. Review the available maintenance documentation system that the fleet uses with the NEV manufacturer thoroughly, as what is available may influence the purchase decision or whether vehicle maintenance is performed using in-house mechanics or contract service.

\subsubsection{Batteries}

If there is currently an in-house maintenance operation at the fleet manager's facility, then many of the following requirements and recommendations will likely be in place. If no maintenance is currently taking place, then there are many requirements and guidelines that need to be implemented when working with batteries. The following general guidelines should be followed at all times to ensure the health and safety of all personnel. 
7.2.5.1 Stored Batteries. Ignition sources should not be allowed within a minimum of 25 feet of any stored batteries. This includes welding, burning, brazing, smoking, and spark-producing equipment such as switches and motors with brushes. The quantity of stored batteries and the maximum time they may be stored are regulated under the Resource Conservation and Recovery Act, and Environmental Protection Agency regulations. ${ }^{\text {h }}$ These regulations should be consulted prior to establishing battery storage methods.

7.2.5.2 Personnel Safety. Personal protective equipment must be used while conducting normal FLA battery/battery system operations and maintenance. This includes:

- $\quad$ Face shield or eye goggles (should be worn at all times while around exposed battery modules)

- $\quad$ Rubber gloves

- $\quad$ Acid/caustic-resistant clothing

- $\quad$ Acid/caustic-resistant footwear.

Personal protective equipment requirements are regulated by the Occupational Safety and Health Administration. ${ }^{\mathrm{i}}$ Other equipment for handling acid and caustic solutions should be used in accordance with each manufacturer's instructions and recommendations and Material Safety Data Sheets (MSDSs). ${ }^{j}$

An eye wash station must be available in close proximity to all areas where FLA battery service is performed. The eye wash station must be capable of providing sufficient irrigation of the eyes should it be needed (electrolyte in the eyes) and must be maintained in accordance with Occupational Safety and Health Administration requirements. ${ }^{\mathrm{k}}$

7.2.5.3 Maintenance procedures. The vehicle manufacturer should provide documentation that outlines all preventative maintenance procedures for the NEV batteries.

7.2.5.4 FLA Batteries. FLA batteries will require regular maintenance, including watering, cleaning, and inspection. Refer to the owners' manual for proper maintenance procedures.

7.2.5.5 VRLA Batteries. VRLA batteries require only occasional inspection. Refer to the owners' manual for proper maintenance procedures.

7.2.5.6 Battery Transportation and Storage. Specific Department of Transportation and Environmental Protection Agency regulations govern transportation and storage of batteries. FLA batteries are considered hazardous material, must be properly stored in approved containers, and require additional paperwork when transporting. VRLA batteries are not considered hazardous material and require no special handling or storage procedures. In all cases, it is a good idea to obtain the MSDS for all batteries in the NEV fleet. The MSDS is produced by the battery manufacturer and should be supplied by the vehicle manufacture. It specifies the materials used in the batteries and any special care required.

\footnotetext{
${ }^{\mathrm{h}}$ See 40 CFR 273.9 for Small Quantity Handler of Universal Waste definition, 40 CFR 273.15 for storage time constraints, and 40 CRF 273 for complete discussion of waste storage.

${ }^{\text {i }} 29$ CRF 1910 parts $132,133,136,137$, and 138

j 29 CFR 1910.1200, Occupational Safety and Health Administration

${ }^{k}$ OSHA General Industry Safety Orders (Section 5162)
} 
MSDS paperwork is always required for transporting FLA batteries and often is required for VRLA batteries.

7.2.5.7 Recycling/Disposal Considerations. The recycling and disposal of batteries should be described in the vehicle owners' manual. The vehicle owner is responsible for properly recycling and disposing of the batteries. All batteries must be recycled through a reputable recycler. Never dispose of any NEV battery in the trash.

\subsubsection{Contract Maintenance Onsite}

If the fleet operator intends to use contract services for maintenance of the NEVs onsite, all of the above listed issues apply to the onsite facilities the contractors will utilize, and to the procedures and safety requirements which they follow.

\subsubsection{Contract Maintenance Offsite}

Where vehicle maintenance is contracted by a local service company and the vehicles are repaired offsite (except for small incidental repairs), there are a few items to consider when negotiating the maintenance contract, such as:

- $\quad$ Spare Parts: It's important to define which party is responsible for spare parts and understanding availability.

- Transportation: Unique to low-speed vehicles, transportation on a flat bed or trailer must be used.

- Maintenance Manuals: The contractor will require a complete set of maintenance manuals in order to properly service the NEVs. 


\section{OPERATOR TRAINING}

\subsection{Driving}

Operator training is critical to the successful deployment of NEVs. Electric vehicles in general operate substantially different than internal combustion engine vehicles, and a few minutes of training with each operator will be well worth the time spent.

\subsubsection{Range}

In most cases, NEVs operate entirely off a lead-acid battery pack, typically at either 48 or $72 \mathrm{~V}$. Vehicle driving range can vary from 15 to $30+$ miles depending on driving conditions (e.g., temperature, flat or hilly terrain), age of the batteries, payload, and operator variations.

\subsubsection{Extreme Climates}

Batteries are typically rated at a temperature of $77^{\circ} \mathrm{F}\left(25^{\circ} \mathrm{C}\right)$ and are reduced in capacity for every degree below that. NEV batteries lose $1-2 \%$ capacity for every $1^{\circ} \mathrm{F}$ in battery temperature below $77^{\circ} \mathrm{F}$. This loss in capacity increases significantly below $40^{\circ} \mathrm{F}$, with complete capacity loss at freezing temperatures. Fortunately, batteries have a large thermal mass and even in low ambient temperatures may stay warm for several hours if the NEV is parked in an unheated area.

\subsubsection{Terrain and Payload}

Terrain and payload have a significant impact on the range of NEVs. Range can vary by as much as $50 \%$ for a hilly terrain or if a NEV is heavily loaded with passengers or cargo. It's important to plan trips carefully and be aware of available charging locations and opportunities.

\subsection{Gauges and Controls}

\subsubsection{Gauges}

Most NEVs carry a very basic gauge package that includes a speedometer, odometer, turn-signal lights, SOC indication, and charging indicator. Figures 13A-B, 14A-B, and 15A-B show examples of various NEV manufacturers' gauges.

The SOC gauge is provided only to give the user a general sense of the capacity left in the battery pack. With a multitude of variables impacting the range of an NEV, it is very difficult to accurately depict what capacity is available. Therefore, it is generally a good practice to not let the battery SOC go lower than 30\%. Discharging the batteries too low can be harmful to the life of the battery pack and may leave the operator stranded with a disabled and possibly damaged vehicle. 

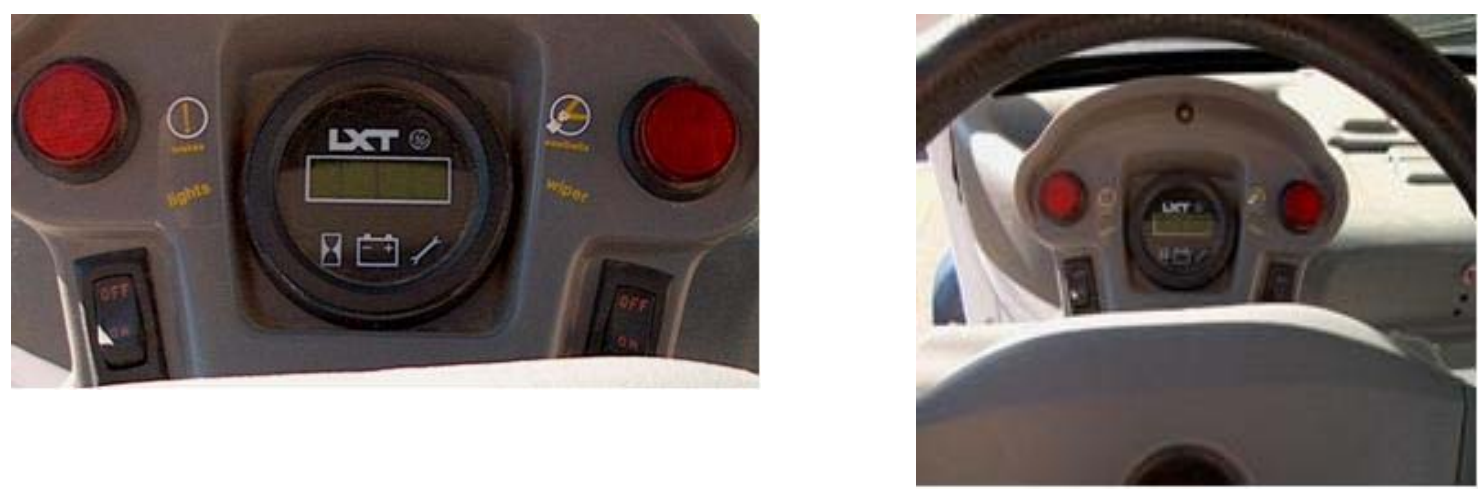

Figures 13A-B. GEM NEV dash with turn signal indicators, and an all-in-one digital display for speedometer, odometer, and battery SOC.
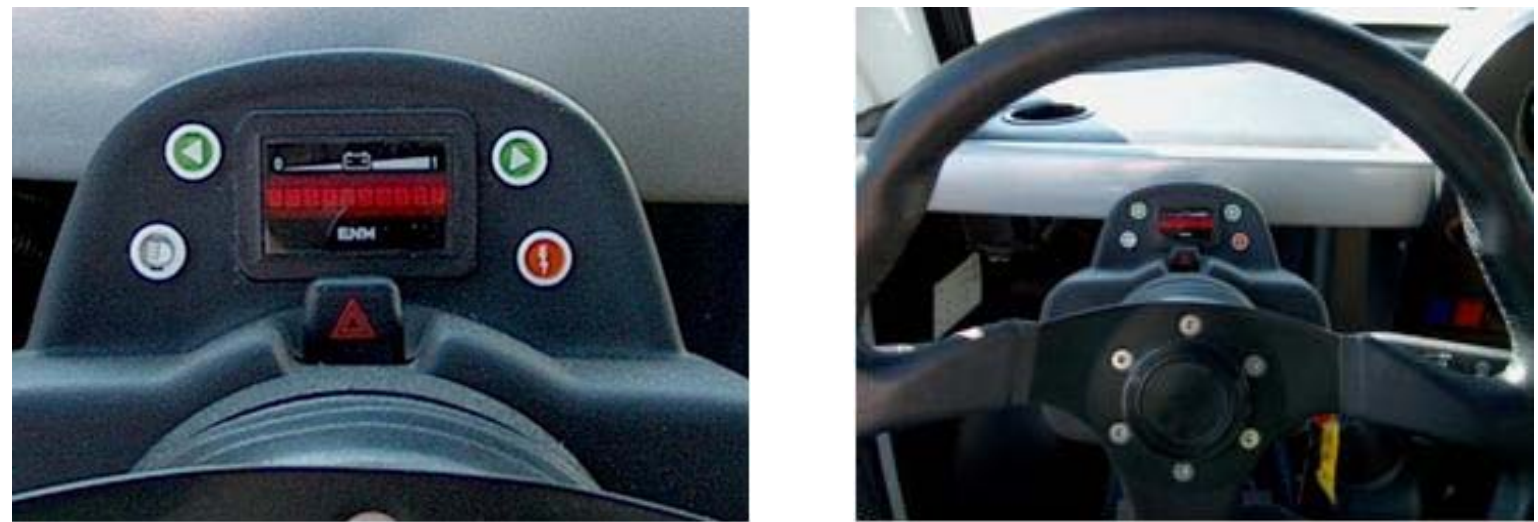

Figures 14A-B. Dynasty NEV SOC gauge, directional signals, high-beam indicator, charging indicator, and emergency flashers.
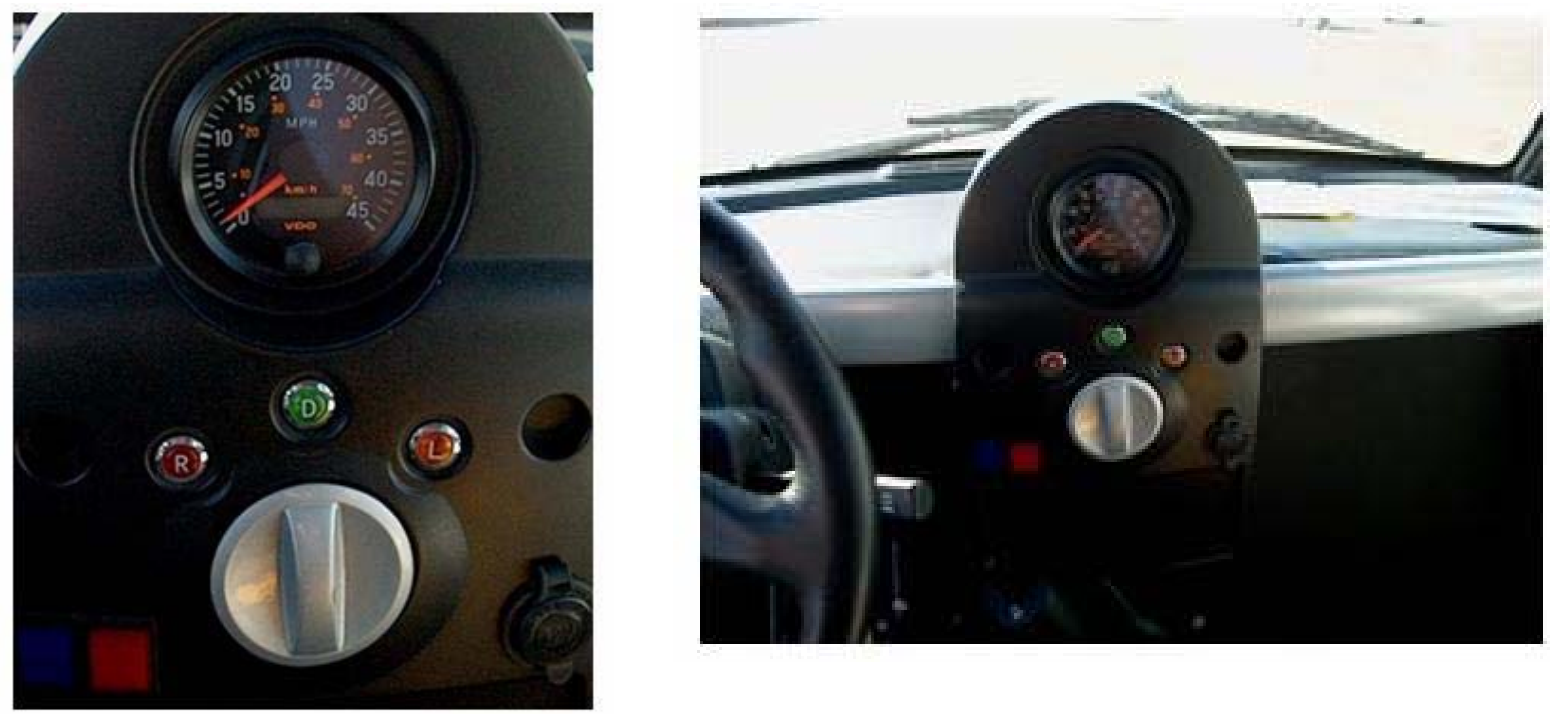

Figures 15A-B. Dynasty NEV center dash displaying the speedometer, odometer, gear selection, and heater/blower controls. 


\subsubsection{Controls}

The controls for NEVs are typically similar to on-road highway vehicles with a few exceptions. Most NEVs offer both a "Road" or "Drive" mode, and a "Turf" or "Low" speed mode as shown in Figures 16A-B. Because these vehicles are usually allowed on golf courses as well as the roads, when the turf or low mode is selected the top speed is limited to 12 to $15 \mathrm{mph}$, similar to a standard golf cart.

Other controls, such as turn signals, lights, horn, etc., are very similar to typical internal combustion vehicles.

\subsubsection{Regenerative Braking}

Most NEVs are equipped with a braking system that recaptures a small percentage of energy through the motor-controller system during braking and coasting. It's best to have long coast-downs and soft braking to maximize the use of regenerative braking and minimize the use of friction braking.

\subsubsection{Charging (Slow, Fast)}

There are two typical types of chargers available for NEVs, the onboard $120 \mathrm{~V}$ charger and an offboard fast charger. Figures 17, 18, and 19A-B show the typical locations for the $120 \mathrm{~V}$ inlet, and Figures 20A-B, 21, and 22A-B show various locations for fast charging.
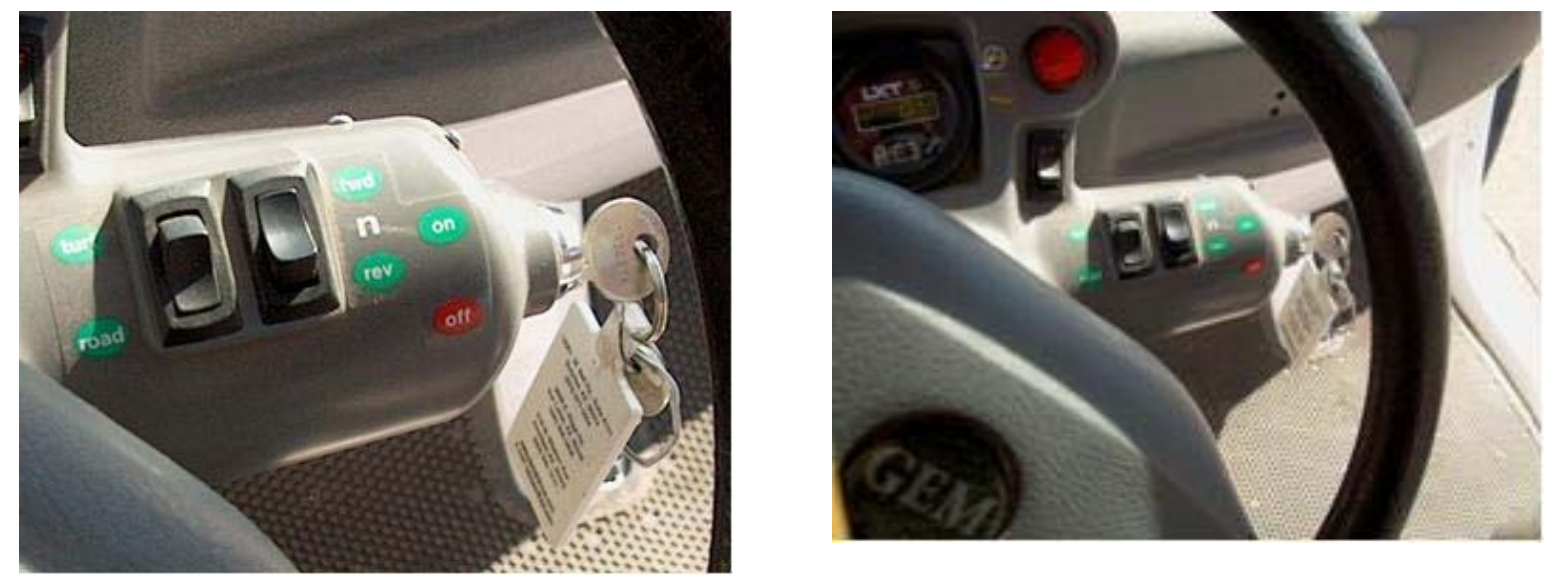

Figures 16A-B. GEM—-turf/road selection and forward/reverse selection.

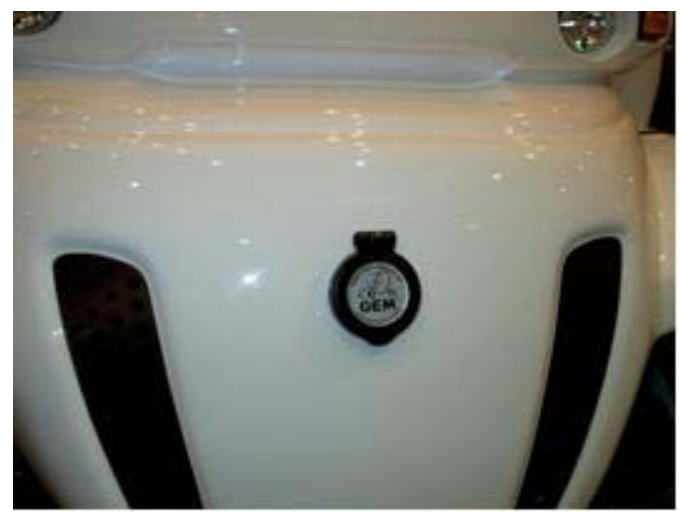

Figure 17. 2003 GEM-120 V inlet located in front hood under the GEM emblem. 


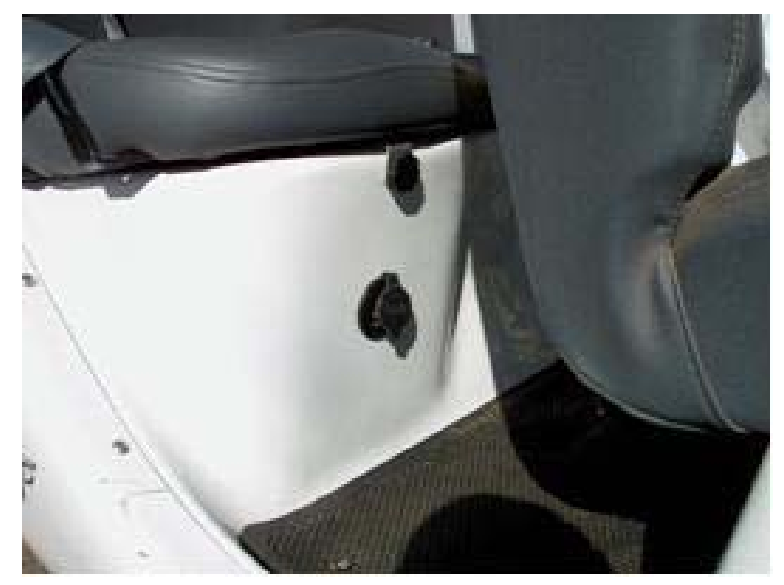

Figure 18. 2002 GEM 4-Passenger $120 \mathrm{~V}$ inlet located below the rear passenger side seat.
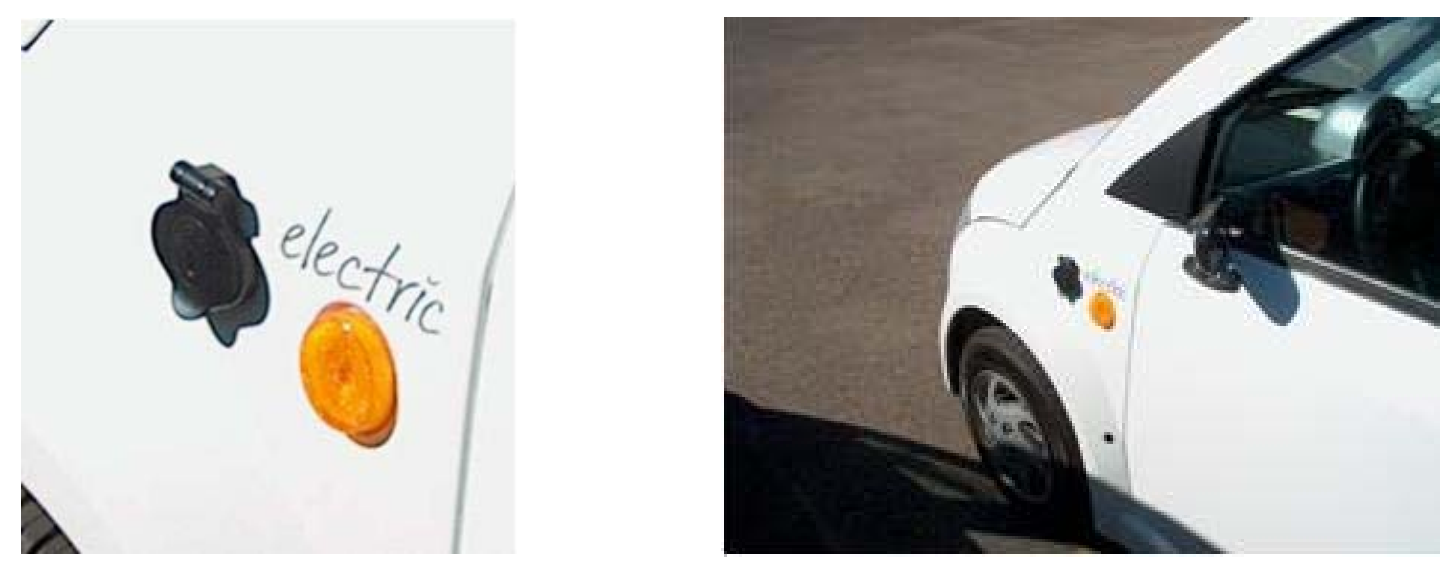

Figures 19A-B. Dynasty “It" $120 \mathrm{~V}$ inlet located on the driver's side front quarter panel.
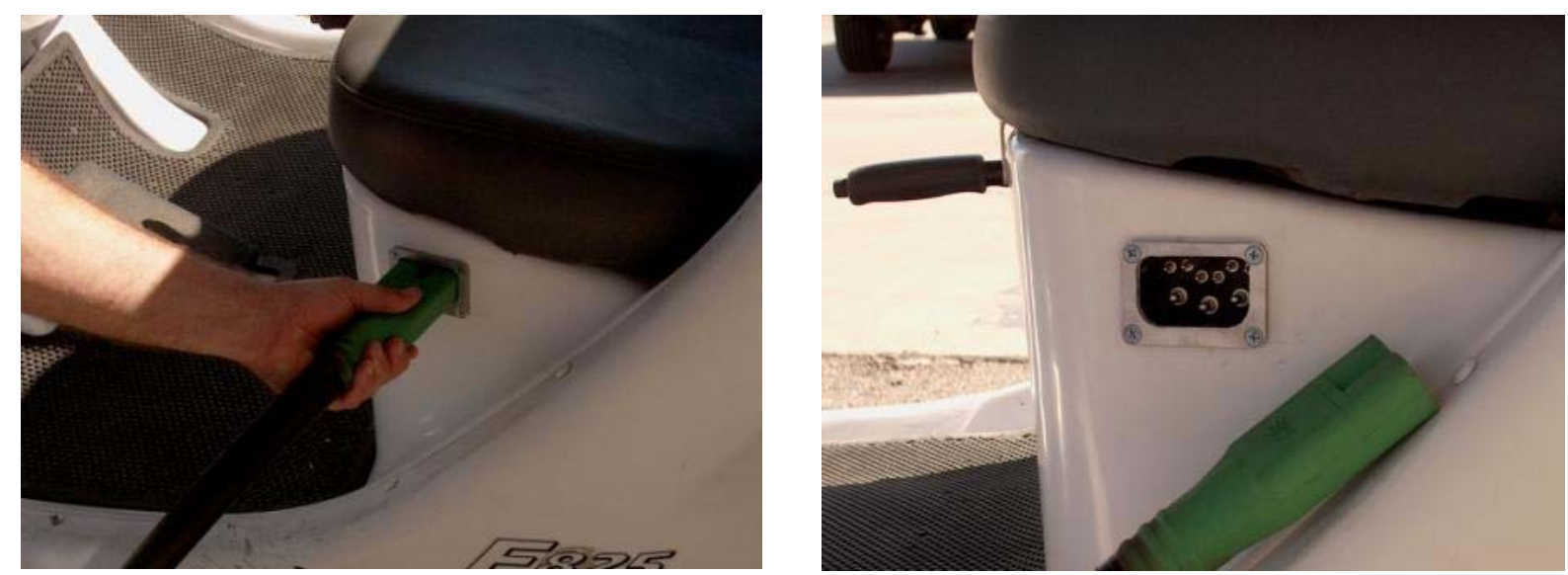

Figures 20A-B. GEM fast charge inlet installed on side of front seat support. 


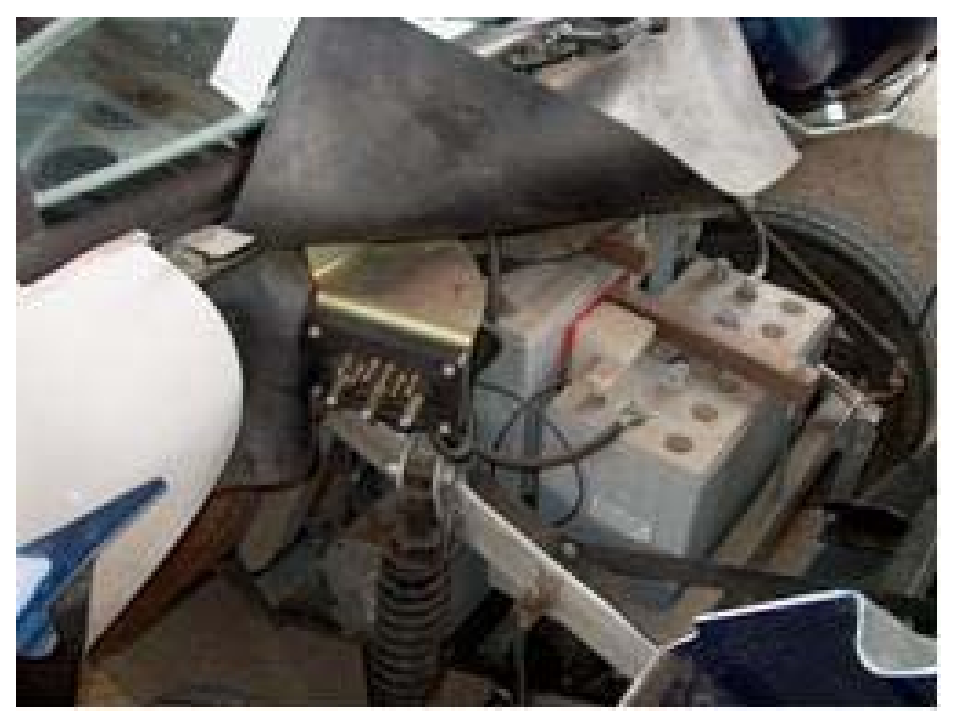

Figure 21. GEM fast charge inlet installed under the front hood.
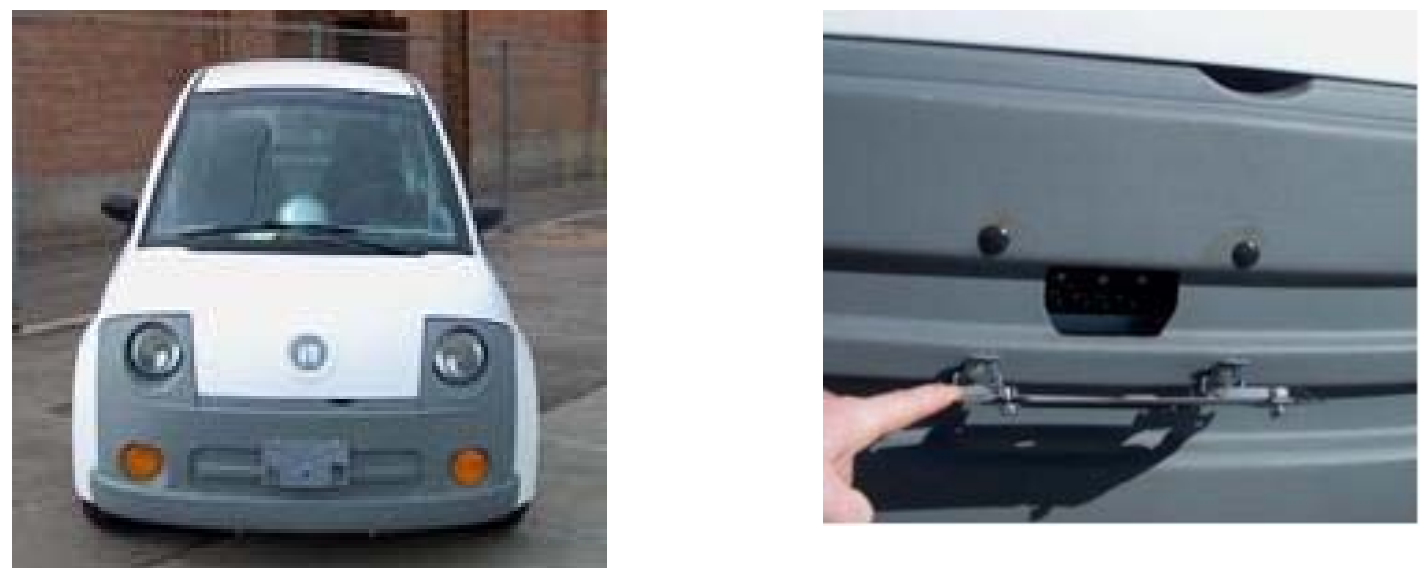

Figures 22A-B. Dynasty front fast charge inlet installed behind the license plate. 


\section{OPERATOR FLEET REPORTING \\ 9.1 Mileage and Maintenance Log}

Implementing some basic fleet reporting is an important step to successfully operating a NEV fleet. It is recommended that monthly vehicle mileage logs and maintenance logs be maintained in order to track any major repairs and the watering schedule. Like any fleet, proper maintenance and a good understanding of how the vehicles are used on a daily basis are critical to a successful operation. Example vehicle mileage and maintenance logs are provided in Appendixes 5 and 6.

\subsection{Operator's Survey}

Another useful tool can be the implementation of an operator's survey. Any feedback from the users, both good and bad, can be very helpful in understanding how well the fleet is running and what improvements can be made. A sample operator survey is provided in Appendix 7. 


\section{FLEET OPERATING COSTS}

The cost to own a NEV (Ownership Cost) consists of three parts; capital cost, operating cost and maintenance cost. Capital costs consist of the purchase cost of the vehicle less any resale or salvage value at the end of vehicle operation. Operating costs consist of fuel cost (in this case the cost of electricity), the cost of licensing the NEV, and the cost of insurance. Maintenance costs consist of all costs associated with preventative and corrective maintenance of the vehicle.

\subsection{Capital Costs}

The typical capital cost of a NEV ranges from $\$ 7,000$ for an entry-level vehicle with very few options to as high as the mid $\$ 20,000$ s range. The type of vehicle (e.g., 2-passenger, 4-passenger, with or without doors) and an endless list of accessories including such options as DVD players, custom colors, and trim features result in a broad range of initial capital costs for NEVs. Resale price for a NEV has historically been soft because of several manufacturers flooding the market with "free" vehicles. These vehicles are often driven for a short period then placed for resale. The large number of vehicles in the resale market has kept the price of used NEVs depressed. A low mileage (less than 100 miles) GEM 2-passenger NEV trades for approximately $\$ 3,500$.

\subsection{Operating Costs}

Based on the results of Baseline Performance Testing conducted for NEVs, the cost of electricity (fuel cost) for NEVs is expected to be a very small part of the overall cost of owning a NEV. The typical electricity (fuel) cost for a NEV during the Baseline Performance Testing was between $1.5 \notin$ and $2.0 \notin$ per mile for electricity priced at $10.0 \notin$ per $\mathrm{kWh}$. In fleet operations, electricity costs were typically less than $10.0 \notin$ per $\mathrm{kWh}$, reducing electricity costs even further. (See the section Comparing NEV Fuel Costs per Mile for additional discussion on fuel costs).

In the majority of the fleets monitored during this study, vehicles were operated on a closed campus and were not licensed or insured. For vehicles operated on public streets (requiring both licensing and insurance), costs varied considerably. Generally, licensing costs are insignificant ( $\$ 25$ to $\$ 100$ per year). In most instances, an alternative fuel vehicle discount was available for licensing. Insuring a NEV was an opposite situation. Many insurance companies were unwilling to provide coverage for a NEV as there is not a loss-history available to provide a rating. In general, insurance was only available to operators insuring other vehicles, and they added a NEV to their existing policy. Costs varied widely, with some NEV insurance costing as much as $\$ 250$ per month.

\subsection{Maintenance Costs}

Maintenance costs can vary by multiple orders of magnitude. Factors driving maintenance costs include:

- $\quad$ Vehicle mission

- $\quad$ Charging infrastructure availability

- Owner diligence in performing preventative maintenance

- $\quad$ Vehicle manufacturer. 
Contrary to intuition, the more a NEV was operated, the less its operating cost was. Vehicles used as "tools" to complete a work mission were generally maintained more meticulously and operated on a more frequent basis, both which reduce corrective maintenance costs. Vehicles operated infrequently, without exception, suffered from battery problems. The battery problems created reliability issues with the vehicle, which resulted in less frequent use and escalating neglect of the vehicle. In the fleets monitored in this study, vehicles were operated as little as 10 miles per month and as much as 500 miles per month.

The availability of charging infrastructure (both onboard and offboard fast charging) was critical to the success of NEVs operating in fleets. Without sufficient infrastructure, batteries were often left discharged and operated below proper charge levels, both of which rapidly destroyed the batteries. Once battery problems begin to arise, use of the NEV is often abandoned, and the vehicle falls into neglect. The availability of sufficient charge infrastructure allows the NEV to be frequently charged and the battery to be operated at high states of charge. Vehicles operated in this manner experienced good battery life and reliable battery operation. Several fleet vehicles operating under fast charge conditions experienced battery lives over 3 years. The availability of charge infrastructure does not guarantee that operators will actually use the infrastructure and charge their vehicles. Operator training remains a critical requirement.

Regular preventative maintenance is critical to minimizing maintenance costs. The most important preventative task is battery watering. The most common cause of battery failure in the NEV fleets monitored in this study was lack of watering. With battery replacement costs ranging from $\$ 500$ to over $\$ 1,000$ per pack, a single battery pack replacement can drive maintenance costs by several dollars per mile. With proper watering and charging (as discussed above), a battery can be expected to operate for 3 years and 15,000 miles. Therefore, the expected battery maintenance cost is $5 \phi$ per mile assuming a $\$ 750$ battery replacement cost. In reality, however, battery replacements occurred as frequently as twice per year, with operating miles of less than 500 miles per battery pack, resulting in battery maintenance costs of $\$ 1.50$ per mile. Other preventative maintenance costs were only significant for vehicles that were operated frequently and over long distances. Brakes and tires were replaced in monitored fleets on older vehicles as a function of both age and of accumulated miles.

Corrective maintenance requirements were a very strong function of the vehicle manufacturer. The fleets monitored were mostly populated with vehicles manufactured by GEM. Early versions of these vehicles experienced a high incidence of charger failures. From model year 2000 through 2005, GEM used three different charger manufacturers. The first two manufacturers experienced high failure rates, which appear to have been eliminated by the third (current) manufacturer. Failures of DC-DC converters were also common, particularly in vehicles subject to salt environments and wash down. The DC-DC converter is not sealed and frequently fails due to corrosion of its input and output terminals. The charger and controller are expensive relative to the resale value of the NEV, often resulting in the vehicle being scrapped when one of these major components failed. 


\section{COMPARING NEV FUEL COSTS PER MILE}

Modeling fuel (electric or gasoline) costs per mile to operate a vehicle, be it a NEV or an internal combustion engine vehicle, is highly dependant on the assumptions employed, including the propulsion efficiency and the per unit cost of fuel. The following assumptions from published sources are used.

The efficiency at the charger, for the twelve NEVs baseline performance tested by the AVTA that used onboard chargers and conductive connectors, is used for the NEV propulsion efficiency. On average, the 12 NEVs required 152 watt-hours AC per mile traveled (Table 5). The efficiency at the charger measures the electrical energy for which the NEV operator must pay the electric utility. It is measured offboard the vehicle, at the utility and NEV connection.

Table 5. NEV energy efficiency as measured at the charger during NEVAmerica baseline performance testing for the $12 \mathrm{NEVs}$ tested with onboard chargers and conductive connectors. Fact sheets for each $\mathrm{NEV}$, including the Efficiency at the Charger data can be found in Appendix 3. Note that the watt-hours are alternating current.

\begin{tabular}{lclc}
\hline \multicolumn{1}{c}{ NEV Model } & $\begin{array}{c}\text { Efficiency at Charger } \\
\text { (Watt-hours per mile) }\end{array}$ & NEV Model & $\begin{array}{c}\text { Efficiency at Charger } \\
\text { (Watt-hours per mile) }\end{array}$ \\
\hline GEM 2005 4 passenger & 169 & GEM 2002 4 passenger & 141 \\
GEM 2005 2 passenger & 184 & GEM 2002 2 passenger & 125 \\
GEM 2005 short bed & 176 & GEM 2002 long bed & 131 \\
GEM 2005 long bed & 182 & GEM 2002 short bed & 109 \\
Ford/Th!nk 4 passenger & 168 & ParCar 4 passenger & 145 \\
Ford/Th!nk 2 passenger & 163 & ParCar 2 passenger & 133 \\
\hline & Average NEV Energy Efficiency (for all 12 NEVs) & 152 \\
\hline
\end{tabular}

The fuel cost for a NEV to travel 1 mile is also dependant on the cost of electricity. Because NEVs may be charged at many locations, the fuel cost per mile for NEVs is calculated based on the average 2005 electricity rates for three sectors: residential rate -9.42 cents per $\mathrm{kWh}$, commercial rate -8.68 cents per $\mathrm{kWh}$, and industrial rate of 5.57 cents per $\mathrm{kWh}{ }^{1}$

NEVs are often used in a fleet mission to replace an internal combustion engine vehicle. Many times they replace a pickup truck that may have an energy efficiency of less than 20 mpg. However, for the sake of argument, a fleet manager may also be considering using a hybrid electric vehicle instead of a $\mathrm{NEV}$ to replace an internal combustion engine vehicle. Therefore, in addition to assuming the internal combustion engine vehicle options get 20 and $30 \mathrm{mpg}$, a hybrid electric vehicle that is assumed to get $45 \mathrm{mpg}$ (as do the Honda Insight and Toyota Prius in AVTA testing ${ }^{\mathrm{m}}$ ) is also included for comparison.

Recently, the cost for a gallon of gasoline has been highly volatile. A believed conservative gasoline unit cost of $\$ 2.50$ per gallon is used as is the current (as of April 24, 2006) retail cost of \$2.91 per gallon as published by the Energy Information Administration. ${ }^{\text {n }}$

As can be seen in Figure 23, the fuel cost per mile for NEVs is significantly lower than the other hybrid electric vehicle and internal combustion engine vehicle options. However, NEVs may provide less vehicle utility than hybrid electric vehicles and internal combustion engine vehicles. That is, the NEVs

\footnotetext{
${ }^{1}$ Energy Information Administration. Electric Power Monthly. March 2006. DOE/EIA-0226 (2006/03). Table 5.3. Average Retail Price of Electricity to Ultimate Customers: Total by End-Use Sector, 1991 through December 2005.

${ }^{\mathrm{m}}$ See Insight data at: http://avt.inel.gov/pdf/hev/hondainsight ar.pdf and Gen II Toyota Prius data at: http://avt.inel.gov/pdf/hev/04toyotaprius ar.pdf

${ }^{\mathrm{n}}$ The retail cost of gasoline is updated often at the EIA website: http://www.eia.doe.gov As of May 1, 2006, \$2.91 was the listed retail cost per gallon as of April 24, 2006.
} 
may not be able to perform some missions that require carrying greater payloads, traveling off-road, and providing more cabin environmental comforts such as air conditioning. However, if a NEV is capable of meeting mission needs, they can be a very viable economic option for a fleet.

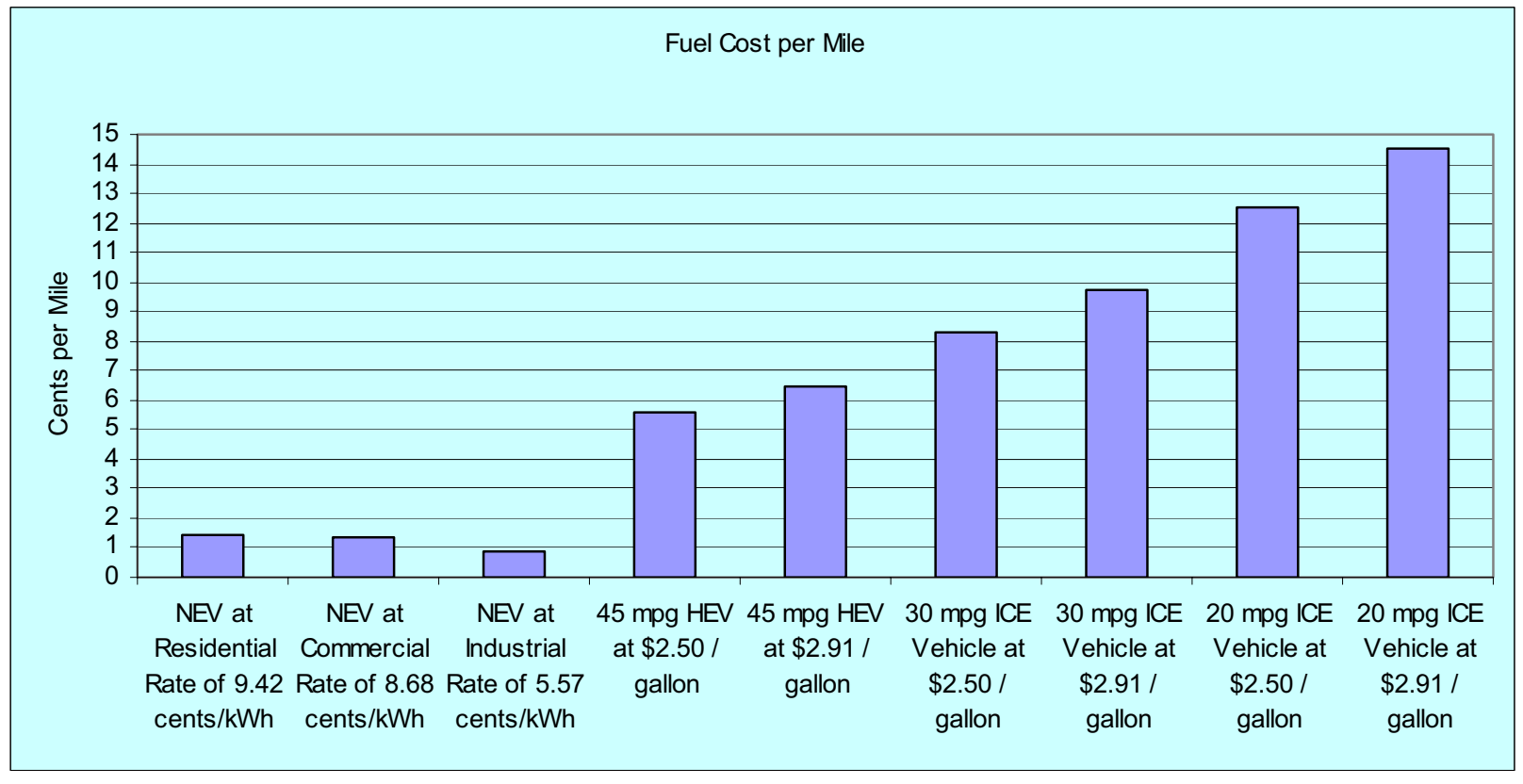

Figure 23. Fuel cost per mile for NEVs, hybrid electric vehicles (HEVs), and internal combustion engine (ICE) vehicles. Three NEV electricity costs are used as are two gasoline costs. 


\title{
APPENDIX 1-FMVSS STANDARD 500
}

\section{TITLE 49--TRANSPORTATION \\ CHAPTER V--NATIONAL HIGHWAY TRAFFIC \\ SAFETY ADMINISTRATION, DEPARTMENT OF TRANSPORTATION}

\author{
PART 571_FEDERAL MOTOR VEHICLE SAFETY STANDARDS--Table of Contents
}

Subpart B - Federal Motor Vehicle Safety Standards

Sec. 571.500 Standard No. 500; Low-speed vehicles.

S1. Scope. This standard specifies requirements for low-speed vehicles.

S2. Purpose. The purpose of this standard is to ensure that low-speed vehicles operated on the public streets, roads, and highways are equipped with the minimum motor vehicle equipment appropriate for motor vehicle safety.

S3. Applicability. This standard applies to low-speed vehicles.

S4. [Reserved]

S5. Requirements.

(a) When tested in accordance with test conditions in S6 and test procedures in S7, the maximum speed attainable in $1.6 \mathrm{~km}$ ( 1 mile) by each low-speed vehicle shall not more than 40 kilometers per hour (25 miles per hour).

(b) Each low-speed vehicle shall be equipped with:

(1) Headlamps,

(2) Front and rear turn signal lamps,

(3) Tail lamps,

(4) Stop lamps,

(5) Reflex reflectors: one red on each side as far to the rear as practicable, and one red on the rear,

(6) An exterior mirror mounted on the driver's side of the vehicle and either an exterior mirror mounted on the passenger's side of the vehicle or an interior mirror,

(7) A parking brake,

(8) A windshield that conforms to the Federal motor vehicle safety standard on glazing materials (49 CFR 571.205).

(9) A VIN that conforms to the requirements of part 565 Vehicle Identification Number of this chapter, and

(10) A Type 1 or Type 2 seat belt assembly conforming to Sec. 571.209 of this part, Federal Motor Vehicle Safety Standard No. 209, Seat belt assemblies, installed at each designated seating position.

S6. General test conditions. Each vehicle must meet the performance limit specified in S5(a) under the following test conditions.

S6.1. Ambient conditions.

S6.1.1. Ambient temperature. The ambient temperature is any temperature between 0 [deg]C (32 $[\mathrm{deg}] \mathrm{F})$ and $40[\mathrm{deg}] \mathrm{C}(104[\mathrm{deg}] \mathrm{F})$.

S6.1.2. Wind speed. The wind speed is not greater than $5 \mathrm{~m} / \mathrm{s}(11.2 \mathrm{mph})$.

S6.2. Road test surface.

S6.2.1. Pavement friction. Unless otherwise specified, the road test surface produces a peak friction coefficient (PFC) of 0.9 when measured using a standard reference test tire that meets the specifications of American Society for Testing and Materials (ASTM) E1136, "Standard Specification for A Radial Standard Reference Test Tire," in accordance with ASTM Method E 1337-90, "Standard Test Method for Determining Longitudinal Peak Braking Coefficient of Paved Surfaces Using a Standard Reference Test 
Tire," at a speed of $64.4 \mathrm{~km} / \mathrm{h}(40.0 \mathrm{mph})$, without water delivery (incorporated by reference; see $49 \mathrm{CFR}$ 571.5).

S6.2.2. Gradient. The test surface has not more than a 1 percent gradient in the direction of testing and not more than a 2 percent gradient perpendicular to the direction of testing.

S6.2.3. Lane width. The lane width is not less than $3.5 \mathrm{~m}(11.5 \mathrm{ft})$.

S6.3. Vehicle conditions.

S6.3.1. The test weight for maximum speed is unloaded vehicle weight plus a mass of $78 \mathrm{~kg}(170$ pounds), including driver and instrumentation.

S6.3.2. No adjustment, repair or replacement of any component is allowed after the start of the first performance test.

S6.3.3. Tire inflation pressure. Cold inflation pressure is not more than the maximum permissible pressure molded on the tire sidewall.

S6.3.4. Break-in. The vehicle completes the manufacturer's recommended break-in agenda as a minimum condition prior to beginning the performance tests.

S6.3.5. Vehicle openings. All vehicle openings (doors, windows, hood, trunk, convertible top, cargo doors, etc.) are closed except as required for instrumentation purposes.

S6.3.6. Battery powered vehicles. Prior to beginning the performance tests, propulsion batteries are at the state of charge recommended by the manufacturer or, if the manufacturer has made no recommendation, at a state of charge of not less than 95 percent. No further charging of any propulsion battery is permissible.

S7. Test procedure. Each vehicle must meet the performance limit specified in S5(a) under the following test procedure. The maximum speed performance is determined by measuring the maximum attainable vehicle speed at any point in a distance of $1.6 \mathrm{~km}$ (1.0 mile) from a standing start and repeated in the opposite direction within 30 minutes.

[63 FR 33216, June 17, 1998, as amended at 68 FR 43972, July 25, 2003. 


\section{APPENDIX 2 - NEV FLEET CHECKLIST}

\begin{tabular}{|c|c|c|}
\hline Description & Reference Section & Completed \\
\hline $\begin{array}{l}\text { Understand the Performance Characteristics of the } \\
\text { various NEVs available on the market }\end{array}$ & 2. NEV Description & \\
\hline Determine your fleet's use characteristics & 4. NEV Fleet Testing & \\
\hline Review the NEV options available & 5. Determination of NEV Mission & \\
\hline $\begin{array}{l}\text { Review the warranty and service provided by the } \\
\text { OEM }\end{array}$ & 5. Determination of NEV Mission & \\
\hline Develop a service plan & 6. NEV Selection & \\
\hline $\begin{array}{l}\text { Review any environmental impacts (e.g., Flooded } \\
\text { batteries) }\end{array}$ & 6. NEV Selection & \\
\hline $\begin{array}{l}\text { Develop a charging infrastructure plan based on the } \\
\text { NEVs daily mission }\end{array}$ & 6. NEV Selection & \\
\hline Assign someone to perform operator training & 7. Infrastructure & \\
\hline Assign someone to maintain vehicle mileage log & 8. Operator Training & \\
\hline Assign someone to maintain the vehicle service log & 8. Operator Training & \\
\hline Assign someone to implement user survey & 8. Operator Training & \\
\hline
\end{tabular}




\section{APPENDIX 3 NEVAMERICA DATA SHEETS}

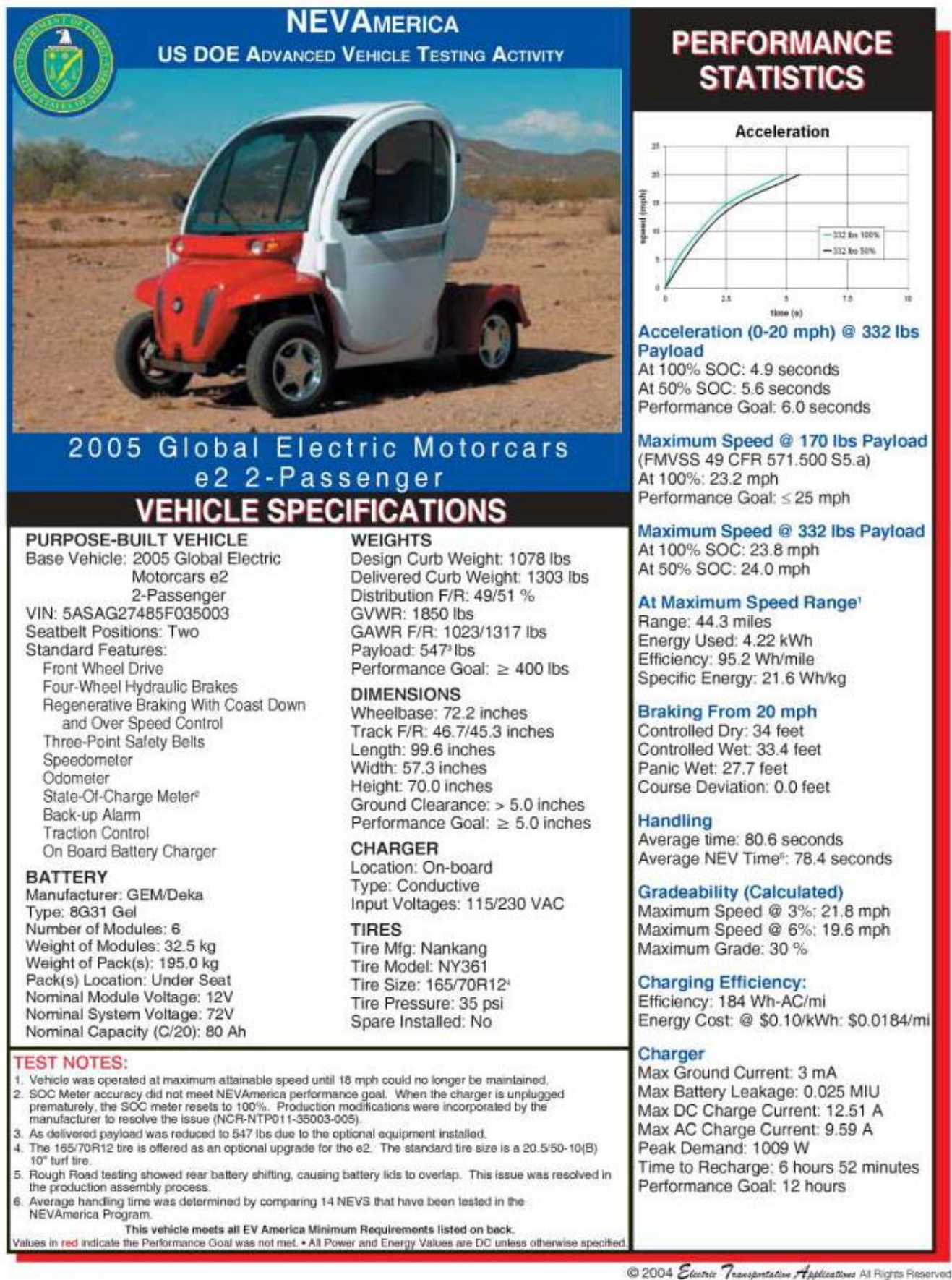




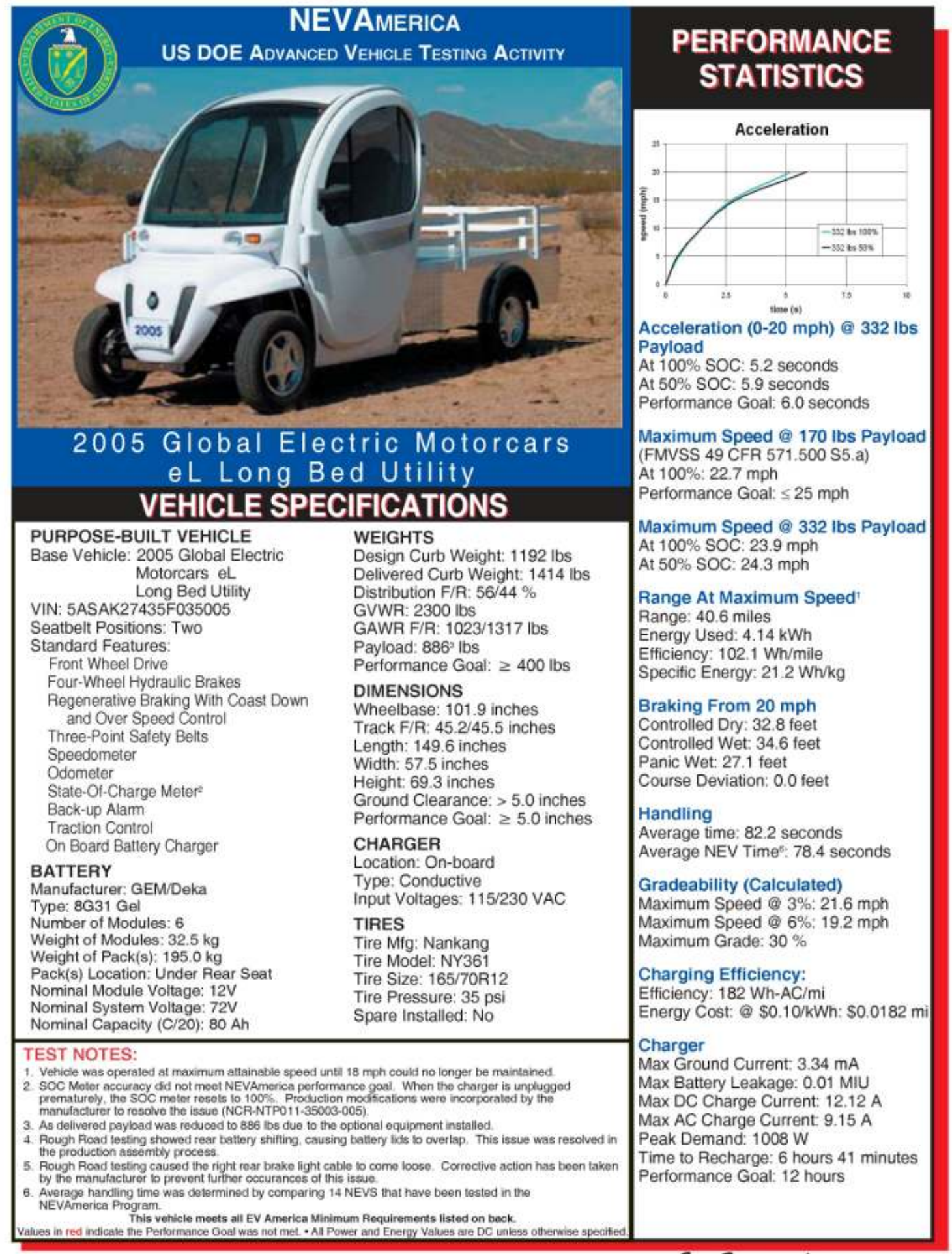




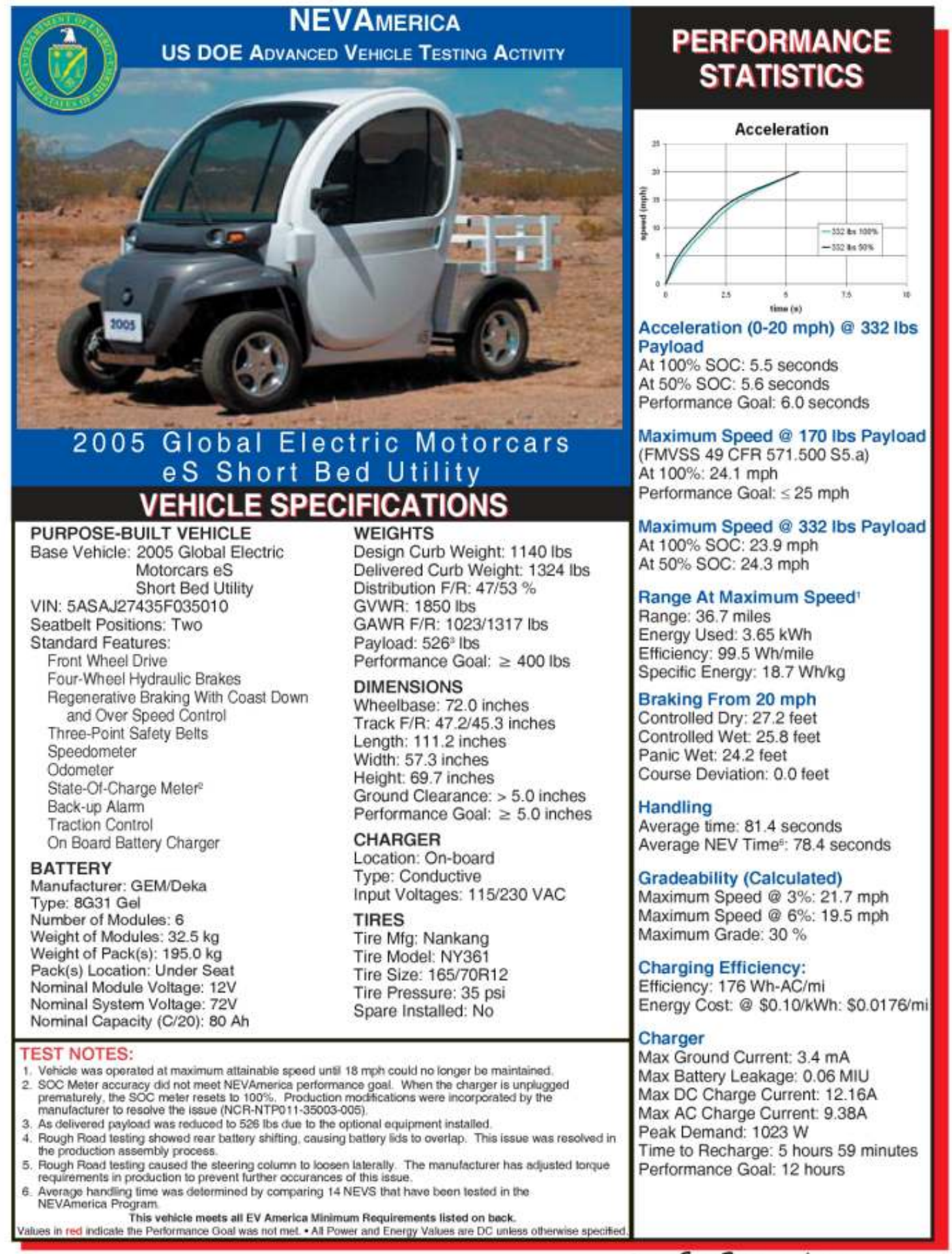




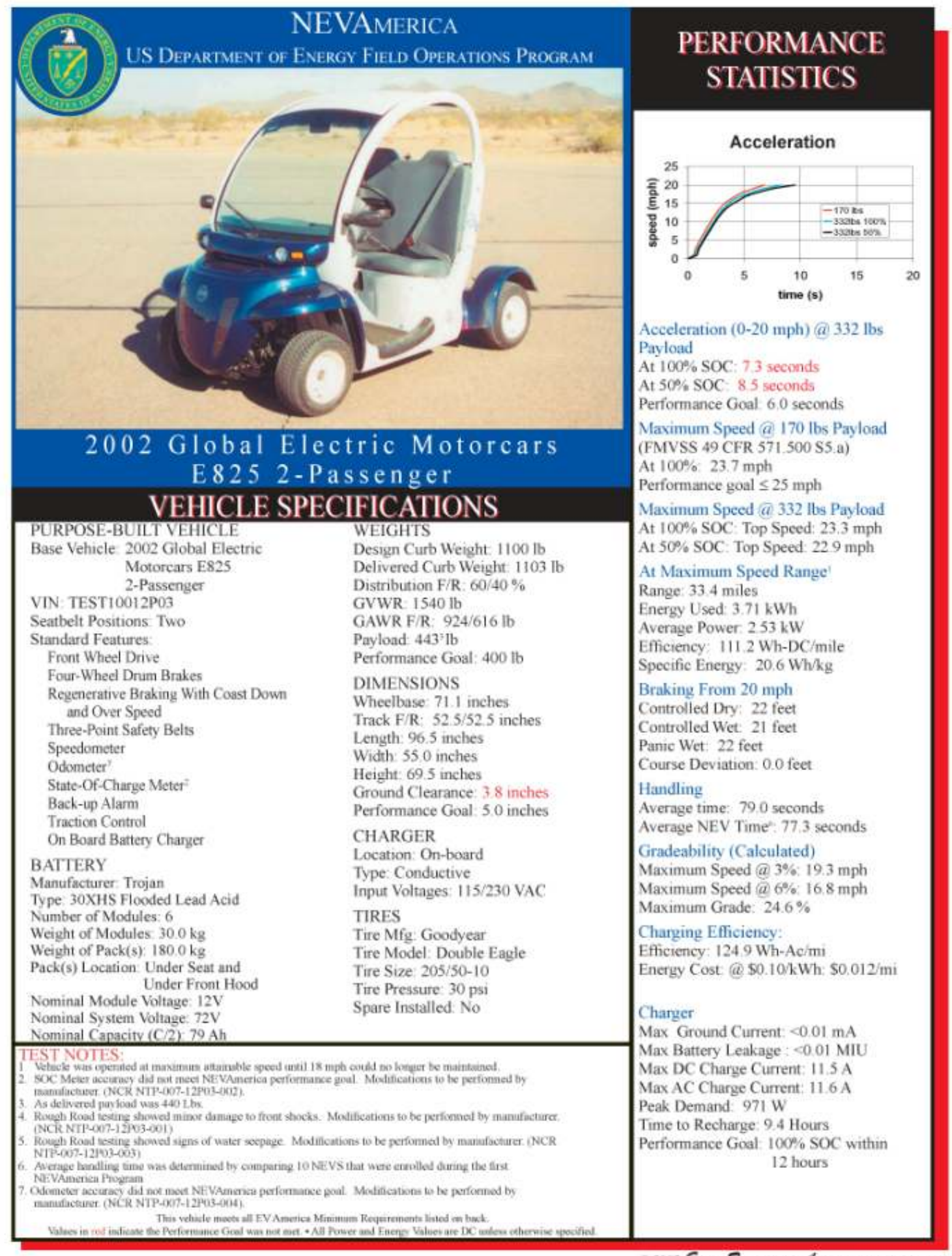

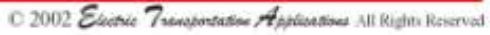




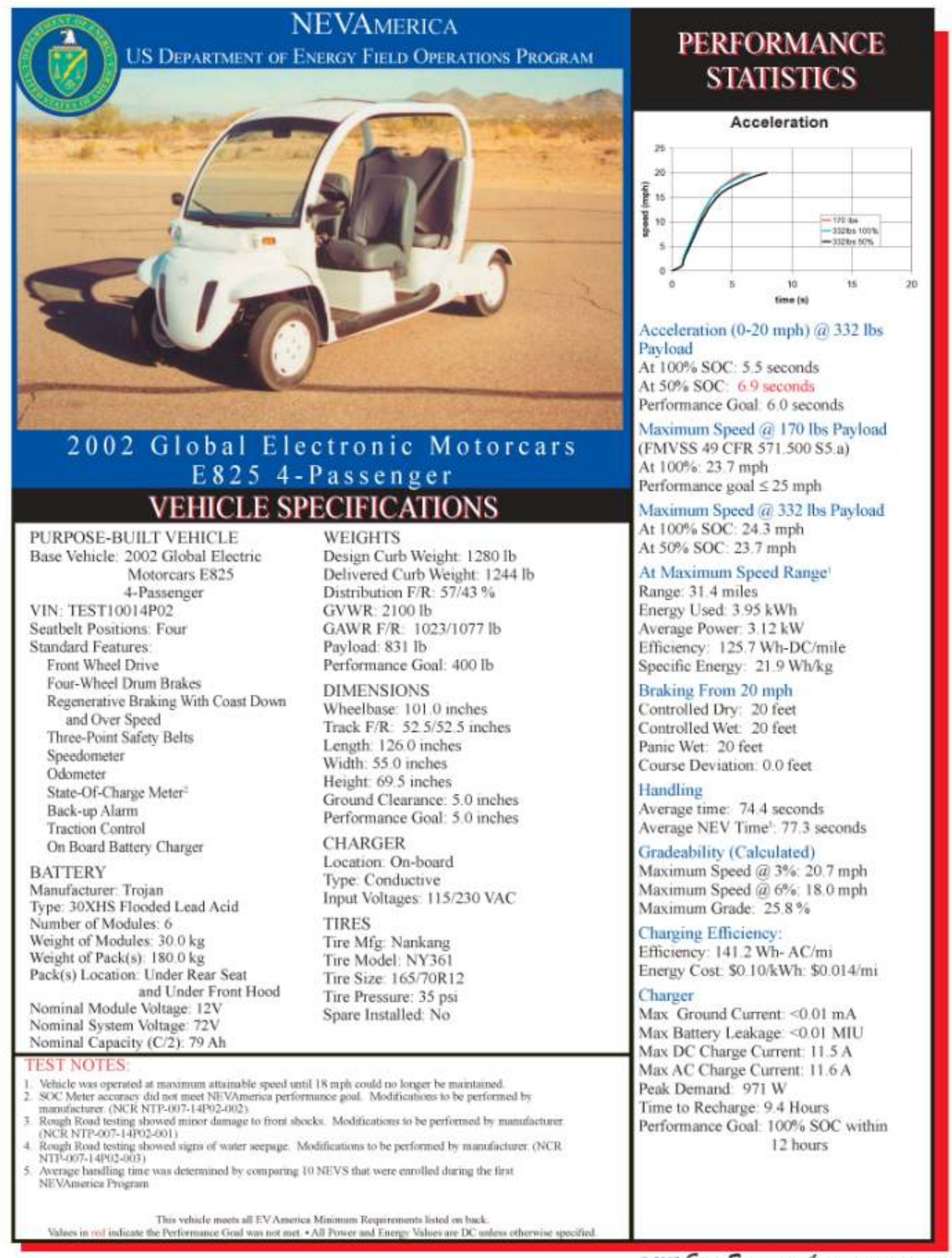

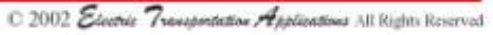




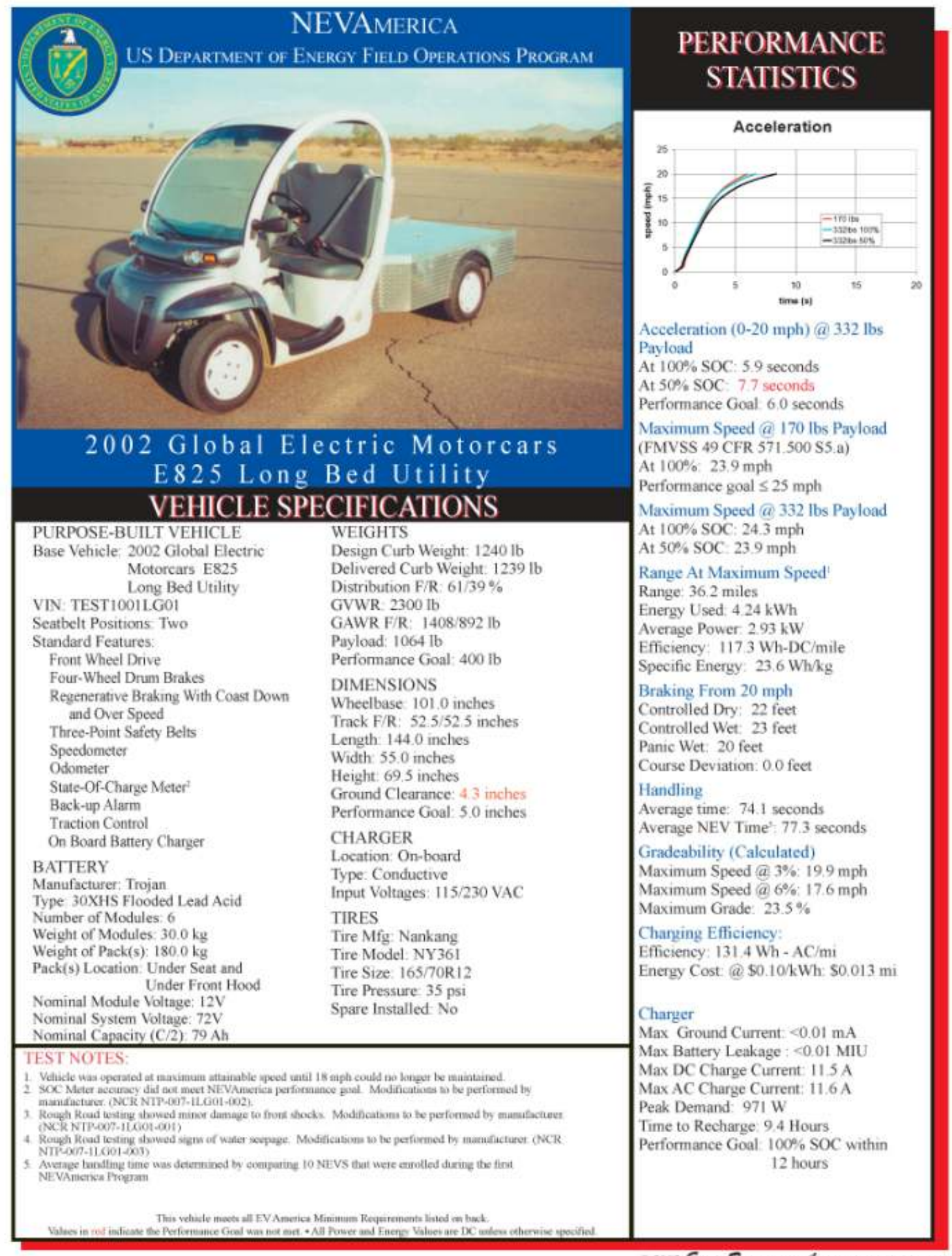

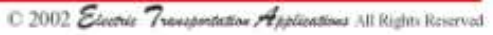




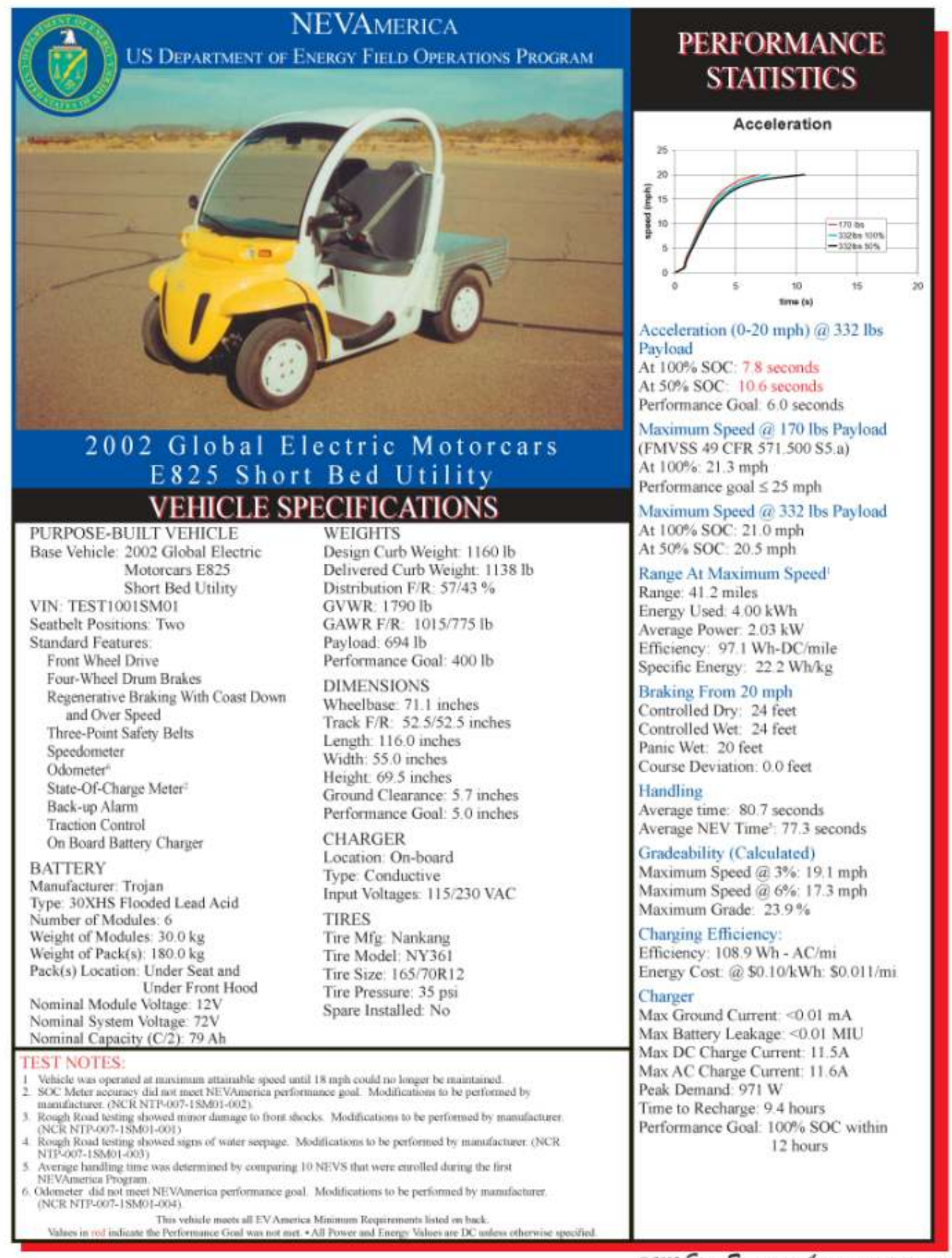

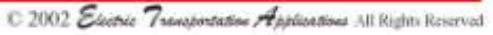




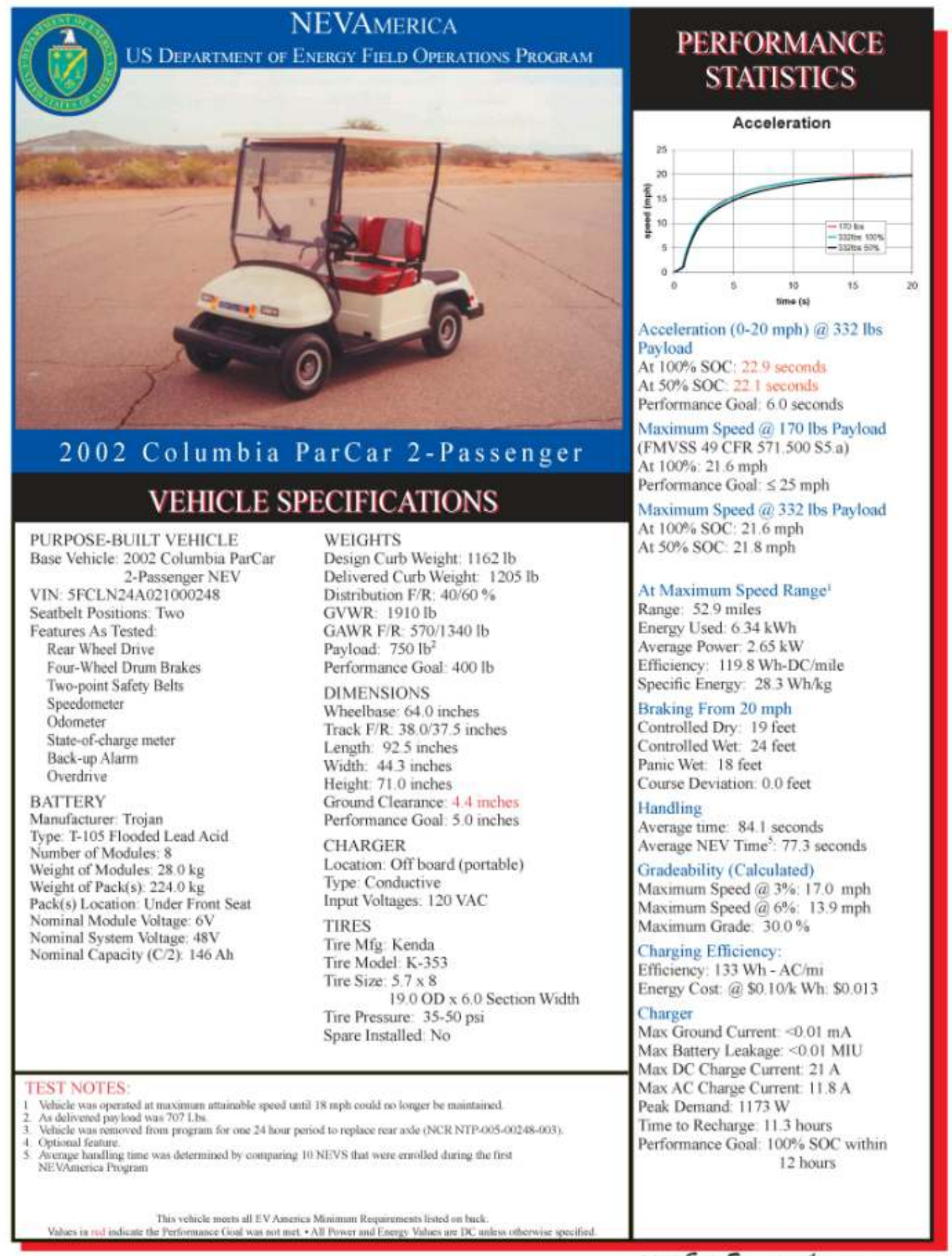




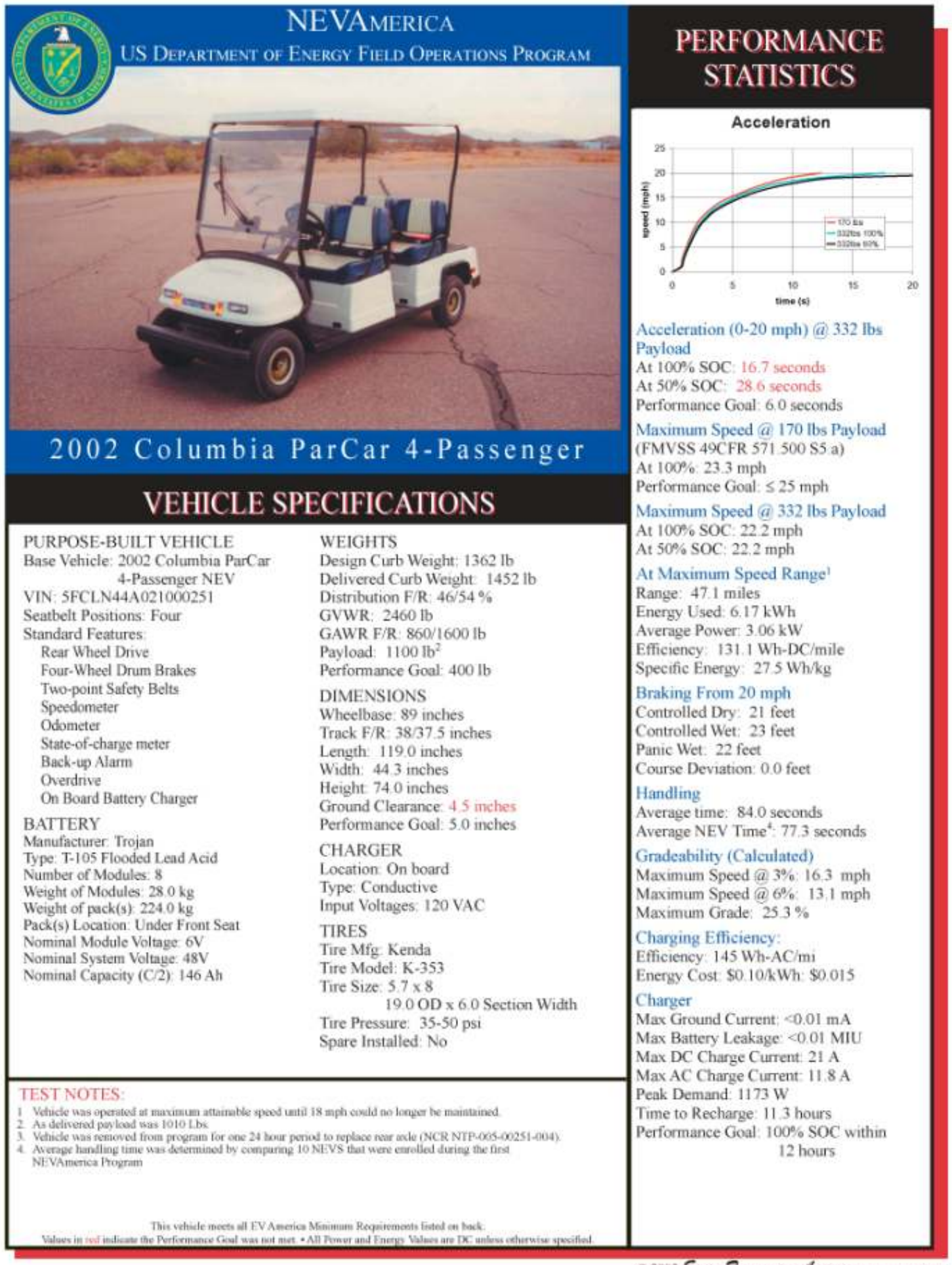

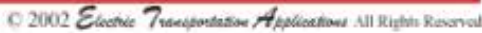




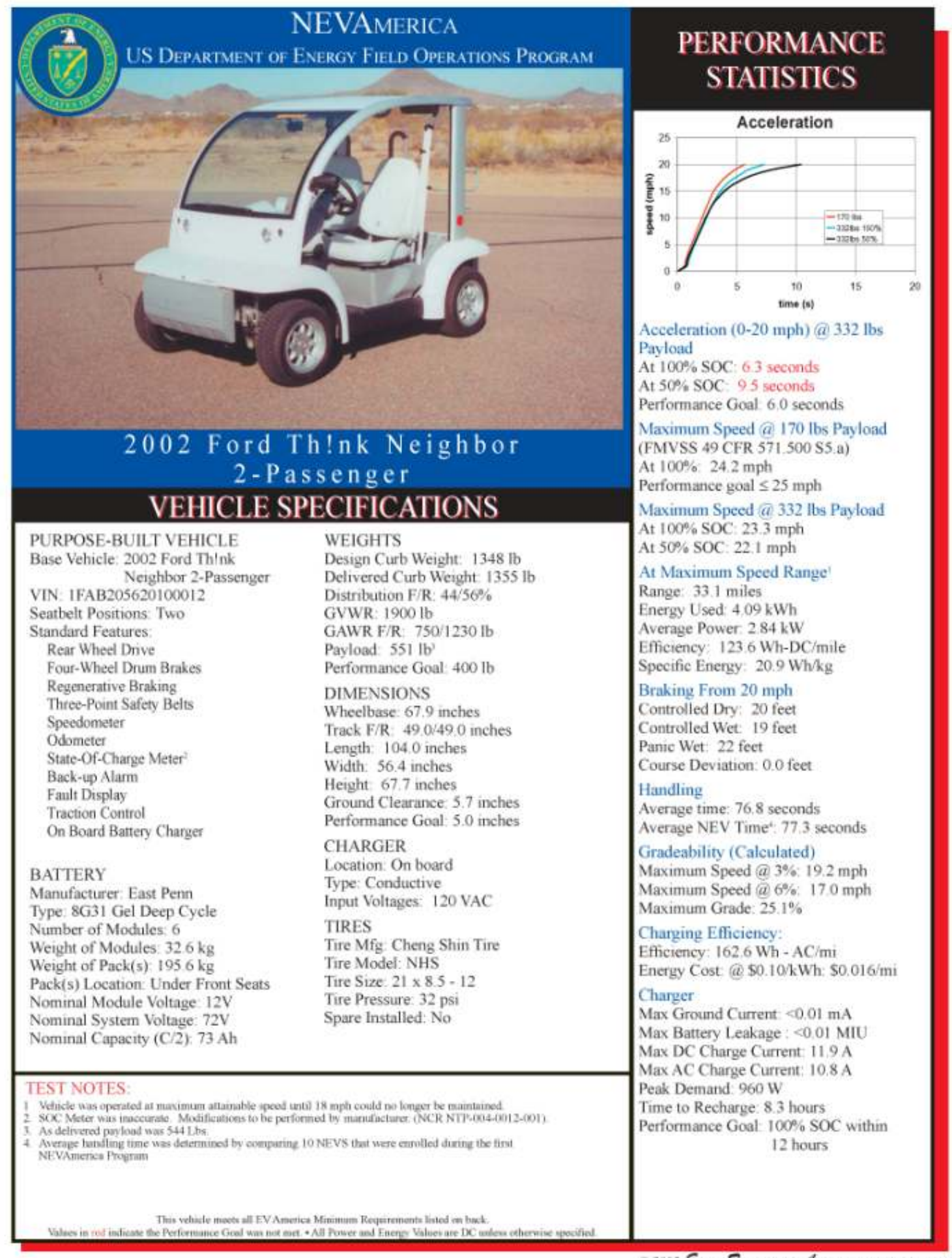

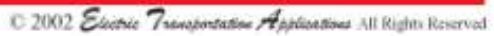




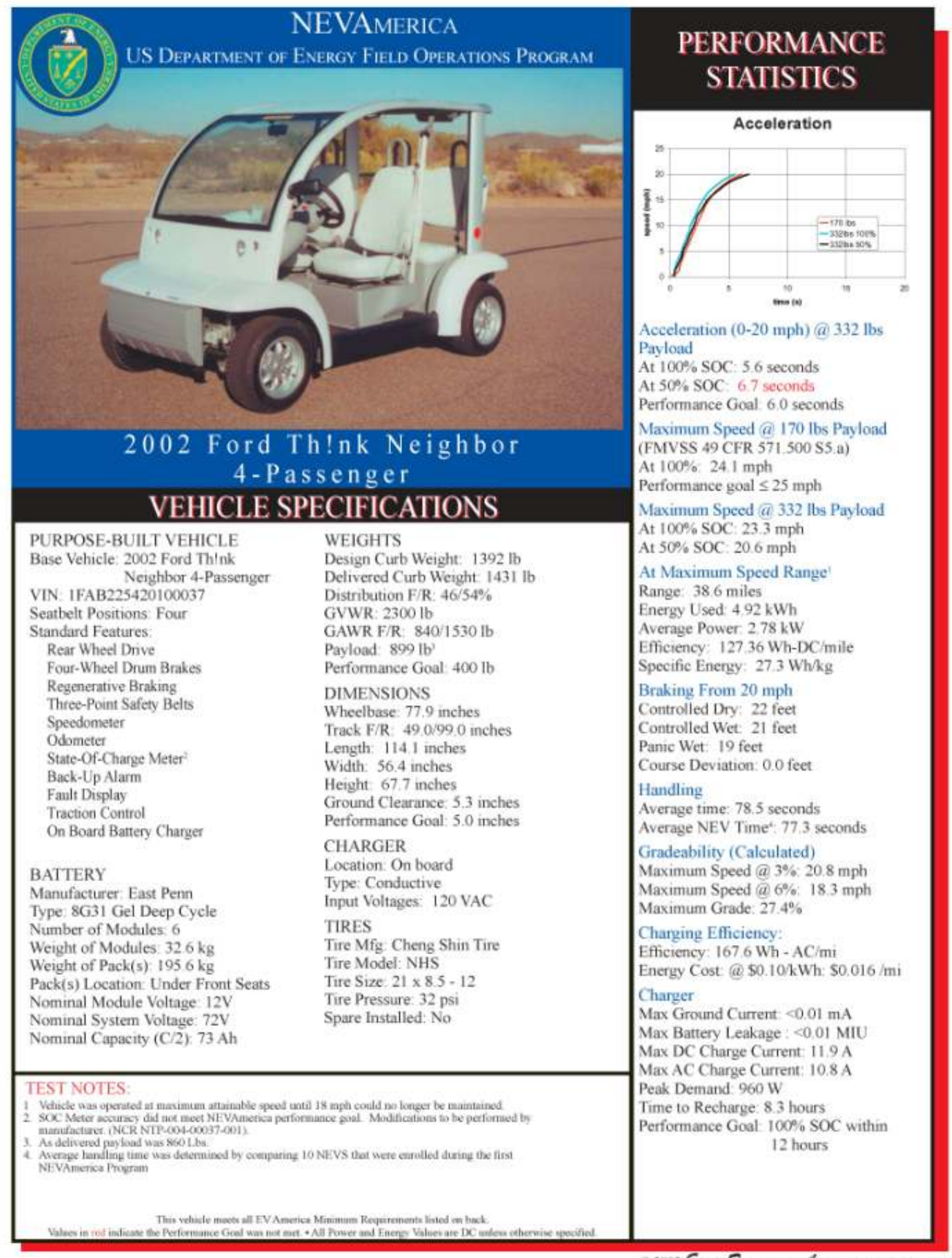

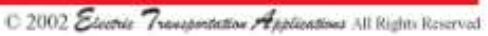




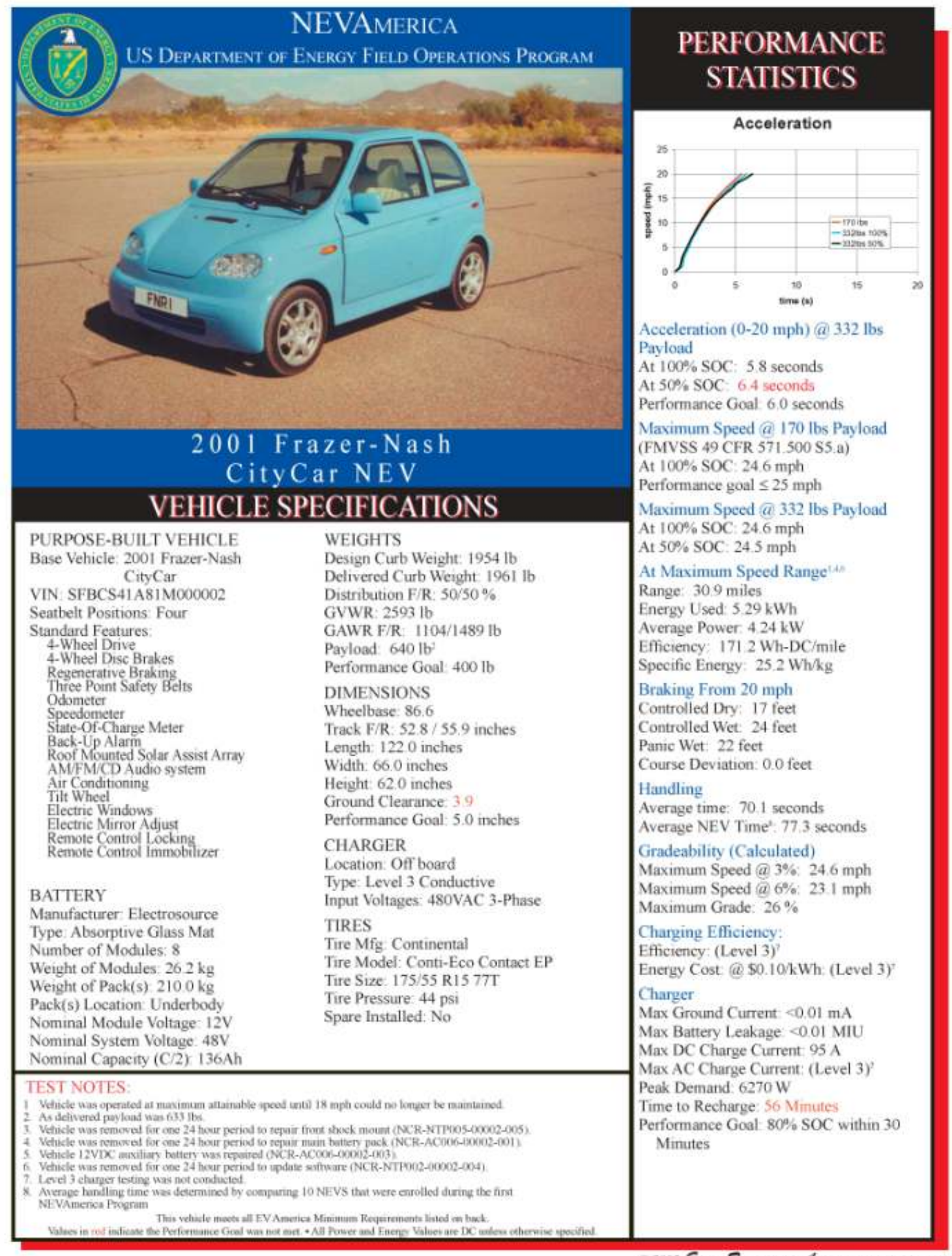

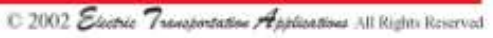




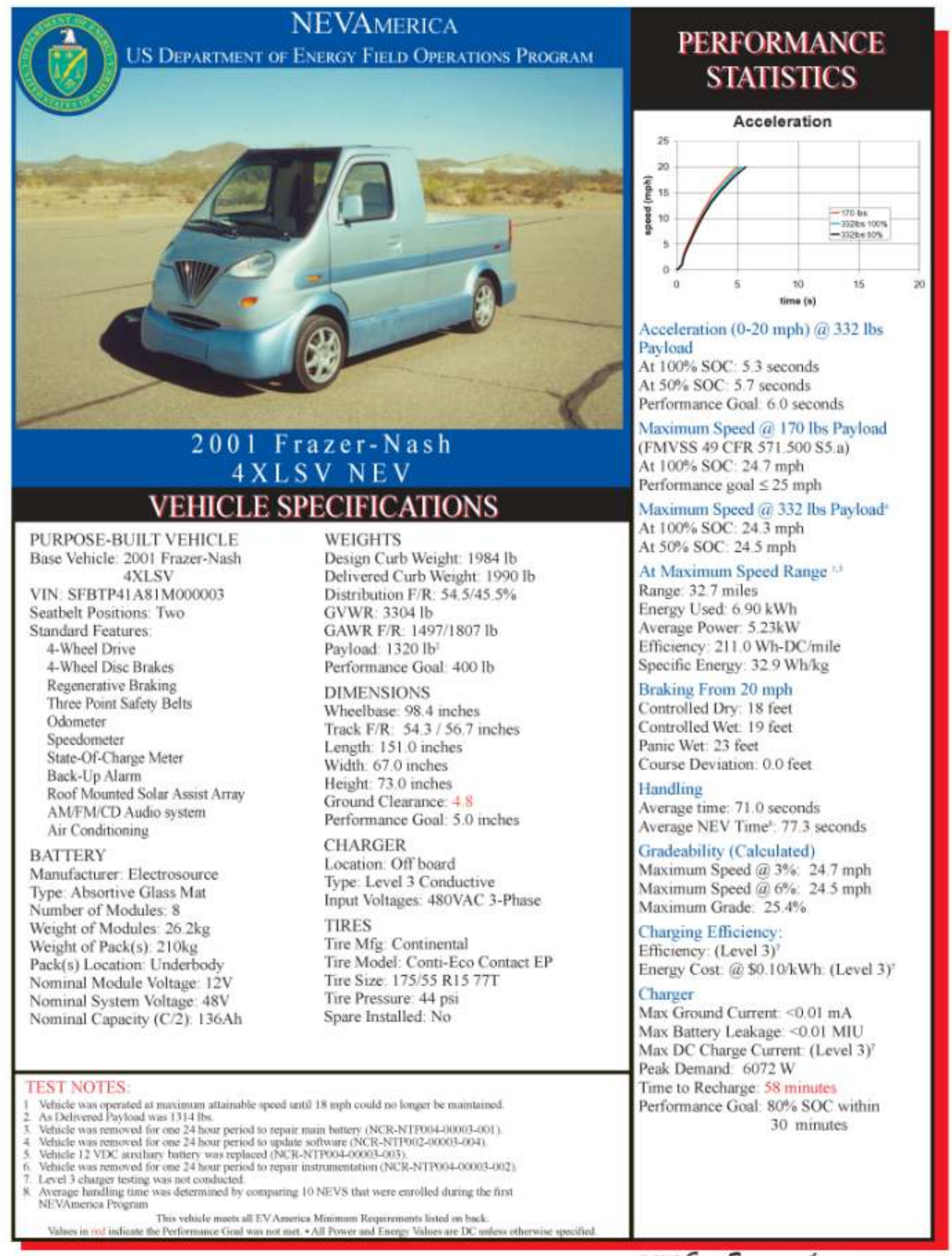

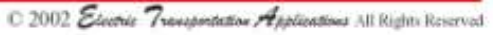




\section{APPENDIX 4 LSV-100 EV OPPORTUNITY CHARGER SPECIFICATIONS}

\begin{tabular}{ll}
\hline Output power & $10 \mathrm{~kW}$ \\
\hline Maximum output current & $100 \mathrm{amps}$ \\
\hline Output voltage options include & $36 \mathrm{~V}, 48 \mathrm{~V}, 72 \mathrm{~V}$, and $96 \mathrm{~V}$ \\
\hline Input power & $208 \mathrm{VAC}, 3$ phase $/ 40$ amps max $(50 \mathrm{~A}$ breaker $)$ \\
& 240 VAC, 1 phase $/ 48$ amps max $(60 \mathrm{~A}$ breaker $)$ \\
\hline Dimensions (H/W/D) & 24 in. $/ 20$ in. $/ 12$ in. \\
\hline Weight & 250 pounds \\
\hline Location/mounting & Indoor/outdoor/wall/pedestal \\
\hline Operating temperature & 0 to $47^{\circ} \mathrm{C}$ \\
\hline Connector & BIW 9-pin \\
\hline Charge times & $<30$ minutes $(30$ to $80 \%$ SOC) \\
\hline
\end{tabular}




\section{APPENDIX 5 SAMPLE VEHICLE MILEAGE LOG FORM}

Month

Year

Vehicle Model:

\begin{tabular}{|c|c|c|c|}
\hline Day of Month & Begin Odometer & Overnight Charged & \# of Opportunity Charges \\
\hline 1 & & $\mathrm{Y} \quad \mathrm{N}$ & 12345 \\
\hline 2 & & $\mathrm{Y} \quad \mathrm{N}$ & 12345 \\
\hline 3 & & $\mathrm{Y} \quad \mathrm{N}$ & 12345 \\
\hline 4 & & Y N & 12345 \\
\hline 5 & & $\mathrm{Y} \quad \mathrm{N}$ & 12345 \\
\hline 6 & & $\mathrm{Y} \quad \mathrm{N}$ & 12345 \\
\hline 7 & & $\mathrm{Y} \quad \mathrm{N}$ & 12345 \\
\hline 8 & & $\mathrm{Y} \quad \mathrm{N}$ & 12345 \\
\hline 9 & & $\mathrm{Y} \quad \mathrm{N}$ & 12345 \\
\hline 10 & & $\mathrm{Y} \quad \mathrm{N}$ & 12345 \\
\hline 11 & & $\mathrm{Y} \quad \mathrm{N}$ & 12345 \\
\hline 12 & & $\mathrm{Y} \quad \mathrm{N}$ & 12345 \\
\hline 13 & & $\mathrm{Y} \quad \mathrm{N}$ & 12345 \\
\hline 14 & & $\mathrm{Y} \quad \mathrm{N}$ & 12345 \\
\hline 15 & & $\mathrm{Y} \quad \mathrm{N}$ & 12345 \\
\hline 16 & & $\mathrm{Y} \quad \mathrm{N}$ & 12345 \\
\hline 17 & & $\mathrm{Y} \quad \mathrm{N}$ & 12345 \\
\hline 18 & & $\mathrm{Y} \quad \mathrm{N}$ & 12345 \\
\hline 19 & & $\mathrm{Y} \quad \mathrm{N}$ & 12345 \\
\hline 20 & & $\mathrm{Y} \quad \mathrm{N}$ & 12345 \\
\hline 21 & & $\mathrm{Y} \quad \mathrm{N}$ & 12345 \\
\hline 22 & & $\mathrm{Y} \quad \mathrm{N}$ & 12345 \\
\hline 23 & & $\mathrm{Y} \quad \mathrm{N}$ & 12345 \\
\hline 24 & & $\mathrm{Y} \quad \mathrm{N}$ & 12345 \\
\hline 25 & & $\mathrm{Y} \quad \mathrm{N}$ & 12345 \\
\hline 26 & & $\mathrm{Y} \quad \mathrm{N}$ & 12345 \\
\hline 27 & & $\mathrm{Y} \quad \mathrm{N}$ & 12345 \\
\hline 28 & & $\mathrm{Y} \quad \mathrm{N}$ & 12345 \\
\hline 29 & & $\mathrm{Y} \quad \mathrm{N}$ & 12345 \\
\hline 30 & & $\mathrm{Y} \quad \mathrm{N}$ & 12345 \\
\hline 31 & & $\mathrm{Y} \quad \mathrm{N}$ & 12345 \\
\hline
\end{tabular}

Instructions for Use of Mileage Log

All drivers should record daily charge information as well as the odometer reading at the beginning of the work day. Documentation should include if the vehicle received overnight charging as well as number of opportunity charges during the worked shift. This will provide the fleet manager sufficient information to evaluate range capability by vehicle. 


\section{APPENDIX 6 SAMPLE VEHICLE MAINTENANCE LOG FORM}

Preventative Maintenance Recommendations for NEV Vehicles

NOTE: Always refer to vehicle handbook for preventative maintenance recommendations for each NEV model.

\begin{tabular}{|c|c|c|c|c|}
\hline & \multirow[b]{2}{*}{ Monthly } & \multirow[b]{2}{*}{ Six Month } & \multirow[b]{2}{*}{ Yearly } \\
\hline & & & & \\
\hline \multirow[t]{2}{*}{ Tires } & Check Air Pressure & $\nabla$ & & \\
\hline & Check Tread Wear & $\nabla$ & & \\
\hline \multirow[t]{3}{*}{ Safety } & Check Horn & $\nabla$ & & \\
\hline & $\begin{array}{l}\text { Check Lights: Front, Rear, Turn signals, } \\
\text { Beacon light (if applicable) }\end{array}$ & $\nabla$ & & \\
\hline & Check Windshield Wipers & $\nabla$ & & \\
\hline Brakes & Adjust Parking Brake & $\sqrt{8}$ & & \\
\hline \multirow[b]{4}{*}{ Traction System } & Check Brake Pads & & & $\bar{\nabla}$ \\
\hline & Check Electrical Connectors & $\vec{\nabla}$ & & \\
\hline & On Board Battery Charger & $\vec{\Delta}$ & & \\
\hline & & & & \\
\hline \multirow[t]{8}{*}{ Battery } & Water Battery (FLA) & $\vec{B}$ & & \\
\hline & Clean Battery Cables & $\vec{\nabla}$ & & \\
\hline & Check Electrical Connectors & 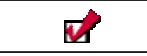 & & \\
\hline & On Board Battery Charger & $\square$ & & \\
\hline & Drive Train Motor & & $\nabla$ & \\
\hline & Drive Differential & & $\sqrt{4}$ & \\
\hline & Lubricate Chassis & & $\nabla$ & \\
\hline & Vehicle Gauges & & $\vec{\Delta}$ & \\
\hline \multirow[t]{3}{*}{$\begin{array}{l}\text { Offboard Fast Charger (if } \\
\text { Applicable) }\end{array}$} & Check Filter (if Applicable) & $\nabla$ & & \\
\hline & Check Cord Connection & $\mathbb{\nabla}$ & & \\
\hline & General Cleaning & & $\vec{\nabla}$ & \\
\hline
\end{tabular}

Maintenance Records for NEV

\begin{tabular}{|l|l|l|l|}
\hline Date & Maintenance/Repair & VIN \# & Cost \\
\hline & & & \\
\hline & & & \\
\hline & & & \\
\hline & & & \\
\hline & & & \\
\hline & & & \\
\hline
\end{tabular}




\section{APPENDIX 7—SAMPLE NEV USER SURVEY}

User Name

NEV Fleet No. recorded.

Circle the Answer that best reflects you opinion and add any additional comments you wish to be

1) How often do you use your NEV?

Daily Several Times a Week Once a Week Couple Times a Month Rarely

Comments

2) What is your primary use of the NEV

People mover Service vehicle Small package delivery

Other, please explain

The NEV meets your daily needs....

Always Almost Always Sometimes Rarely Never

Please explain

4) Rate your available charging infrastructure

Always meets my needs Usually meets my needs Rarely meets my needs Never meet my needs Comments

5) If it Rarely or Never meets your needs, what do you believe would help?

Additional 110 V Outlets Fast Charger(s)

Please explain

6) How do you consider the reliability of your NEV?

Excellent Good Fair Poor Unknown

Comments

7) Any additional comments you wish to make? 